\title{
Fermionization for charge degrees of freedom and bosonization of spin degrees of freedom in the $\mathrm{SU}(2)$ slave-boson theory
}

\author{
Ki-Seok Kim \\ Institute de Physique Théorique, CEA, IPhT, CNRS, URA 2306, F-91191 Gif-sur-Yvette, France
}

(Dated: August 27, 2021)

\begin{abstract}
Fermionizing the charge sector and bosonizing the spin part in the $\mathrm{SU}(2)$ slave-boson theory, we derive an effective field theory for dynamics of doped holes in the antiferromagnetically correlated spin background, where spin fluctuations are described by an $\mathrm{SO}(5)$ Wess-Zumino-Witten (WZW) theory while dynamics of doped holes is characterized by $\mathrm{QED}_{3}$ with a chemical potential term. An important feature of our effective field theory is the coupling term between valance bond fluctuations and doped holes. Considering that valance bond fluctuations are deeply related with monopole excitations of staggered U(1) gauge fields in the bosonic field theory for spin fluctuations, we demonstrate that hole dynamics helps deconfinement of bosonic spinons near the quantum critical point of the $\mathrm{SO}(5)$ WZW theory. We solve this effective field theory in the Eliashberg framework, and find non-Fermi liquid physics in thermodynamics and transport, where $z=3$ criticality with dynamical exponent $z$ plays an important role for hole dynamics. We discuss validity of our field theory, applying it to a doped spin chain and comparing it with the slave-fermion framework. Furthermore, we discuss instability of the anomalous metallic phase against superconductivity and density waves of doped holes, resulting from competition between gauge and valance bond fluctuations.
\end{abstract}

PACS numbers: 71.10.Hf, 74.20.Mn, 75.10.-b, 11.10.Kk

\section{INTRODUCTION}

Doping to an antiferromagnetic Mott insulator has been one of the central interests in modern condensed matter physics, associated with high $\mathrm{T}_{c}$ superconductivity. Since the normal state is extremely anomalous, particulary shown from the absence of quasiparticle excitations near $( \pm \pi, 0)$ and $(0, \pm \pi)$ momentum points 1] and temperature quasi-linear behavior in transport experiments [2] although well defined electron excitations seem to exist near $\left( \pm \frac{\pi}{2}, \pm \frac{\pi}{2}\right)[1,2]$, emergence of rather conventional BCS-type superconductivity from such an abnormal normal state has been a long standing puzzle and still does. Such an anomalous behavior would be surely due to strong correlations between electrons, associated with Mott physics.

Slave-boson approach has been one of the canonical frameworks for study of strongly correlated electrons. In particular, a doped Mott insulator problem was formulated in the slave-boson context, [3] where strong repulsive interactions cause so called the single occupancy constraint naturally imposed in the slave-boson representation, and link variables arise as collective "order parameter" excitations formulated as gauge fields. U(1) slaveboson gauge theory has been enjoyed both intensively and extensively for the doped Mott insulator problem, but such a formulation turns out to have fundamental difficulty for d-wave superconductivity emerging from a doped Mott insulator. [4]

Wen and Lee have developed an SU(2) formulation, which extends the $U(1)$ slave-boson theory to include fluctuations between nearly degenerate U(1) mean-field states, well applicable in underdoped regions. [3] Based on their $\mathrm{SU}(2)$ construction, they could obtain the doping independent decreasing ratio of superfluid weight with a confinement ansatz. In addition, they predicted a special structure of a vortex, which has a staggered flux core. Furthermore, they argued similarity between their staggered flux phase of the SU(2) slave-boson theory and the Gutzwiller projected BCS wave function based on an explicit numerical evaluation.

Although the $\mathrm{SU}(2)$ slave-boson framework has explained many kinds of aspects for high $\mathrm{T}_{c}$ cuprates such as phase diagram, thermodynamics, transport, and etc., [3] antiferromagnetic spin fluctuations are difficult to take into account in this context. High $\mathrm{T}_{c}$ superconductivity is sometimes argued to emerge from the spin liquid phase described by the spin sector of the slave-boson theory in the slave-boson community, but its connection to antiferromagnetism is an important issue since the original problem is doping to an antiferromagnetic Mott insulator instead of doping to the spin liquid one. This motivates us to propose how to introduce antiferromagnetic correlations in the $\mathrm{SU}(2)$ slave-boson framework.

Study of quantum antiferromagnetism associated with high $\mathrm{T}_{c}$ cuprates has been performed in the context of spin liquid. In the bosonic representation of spin the half filled quantum antiferromagnet on the square lattice is described by the $\mathrm{O}(3)$ nonlinear $\sigma$ model, allowing a quantum phase transition from an antiferromagnet to a quantum disordered paramagnet. Bernevig et al. have claimed that although the appropriate off-critical elementary degrees of freedom are given by either spin 1 excitons (gapped paramagnons) in the quantum disordered paramagnet and spin 1 antiferromagnons in the antiferromagnet, at the quantum critical point such excitations should break up into more elementary spin $1 / 2$ excitations usually called spinons. [5] This was challenged by Senthil et al. [6] They argued that since the phase transition in Ref. [5] is supposed to fall into Landau-Ginzburg- 
Wilson paradigm, the spectrum at the quantum critical point should be fully understandable only in terms of spin 1 bosonic degrees of freedom. 7] Senthil et al. proposed, as a possible candidate for a deconfined quantum critical point, a direct quantum phase transition between a Neel antiferromagnet and a valance bond solid state, where one gets spinon condensation in the Neel state while instanton excitations (tunnelling events between energetically degenerate but topologically inequivalent gauge vacua in the $\mathrm{CP}^{1}$ representation of the $\mathrm{O}(3)$ nonlinear $\sigma$ model) should possibly arise in the paramagnetic phase, whose condensation does not allow spinon deconfinement. Senthil et al. demonstrated that such proliferation of instantons is not the case at the quantum critical point, where a topological $\theta$ term usually referred as a Berry phase term makes instantons irrelevant and accordingly, makes it possible to achieve spinon deconfinement.

This proposal motivated direct numerical simulations of various microscopic models to find such an exotic quantum critical point beyond the Landau-GinzburgWilson paradigm. Actually, Sandvik has claimed that such a critical point exists indeed in a modified Heisenberg model with four-spin interactions. [8] Furthermore, he pointed out an important thing, that is, only one length scale seems to exist and accordingly, critical exponents for both staggered spin correlations and valance bond fluctuations are the same as each other, apparently in contrast with the original proposal for deconfined quantum criticality of the bosonic field theory.

Resorting to the fermion representation of spin, $\mathrm{QED}_{3}$ or $\mathrm{QCD}_{3}$ was obtained as an effective field theory at half filling, where its conformal invariant fixed point is allowed in the large $N$ limit, identified with algebraic spin liquid. [9, 10] An important point is that this effective field theory has an enlarged symmetry compared with its microscopic Hamiltonian. $\mathrm{QCD}_{3}$ resulting from the $\pi$ flux ansatz has $\mathrm{Sp}(4) \approx \mathrm{SO}(5)$ while $\mathrm{QED}_{3}$ arising from the staggered flux gauge exhibits $\mathrm{SU}(4)$, where both are basically associated with spin and nodal structures. [9, 10] This enlarged symmetry has important physical implication that symmetry equivalent operators have their same scaling dimension, which should be taken into account for an effective field theory of such composite field variables.

Actually, Tanaka and Hu have derived an effective field theory for competition between antiferromagnetism and valance bond solid.[11] Starting from the $\pi$ flux phase, they obtain $\mathrm{QCD}_{3}$ as its effective field theory although they do not take such gauge fluctuations into account explicitly. Their crucial observation is that the antiferromagnetic order parameter is symmetry equivalent to the valance bond one via chiral rotation in $\mathrm{SO}(5)$. Introducing a chiral rotated mass term with an $\mathrm{SO}(5)$ superspin vector, they could derive an $\mathrm{SO}(5)$ Wess-Zumino-Witten (WZW) theory. Validity of this description is further supported by the fact that the $\mathrm{SO}(5)$ WZW theory can be regarded as a natural extension of the $\mathrm{SO}(4)$ WZW theory for one dimension, [12] which nonabelian bosonization for spin degrees of freedom or equivalently $\mathrm{SU}(2)$ chiral anomaly gives rise to. This discovery is meaningful since it tells a possible connection with the numerical simulation of Sandvik [8] although an appropriate treatment for the topological term is not known, thus true solutions for such an effective field theory are not found. Furthermore, such competition between antiferromagnetism and valance bond solid is argued to be reflected as the checker board pattern in the scanning tunnelling microscopy. 13]

In this paper we follow the same strategy with the study of Tanaka and $\mathrm{Hu}$ for spin degrees of freedom, but away from half filling introducing charge fluctuations based on the $\mathrm{SU}(2)$ slave-boson framework. Two important problems can arise away from half filling. One cautious theorist may concern that a chemical potential term will appear in the Dirac theory of the fermionic spin representation away from half filling, thus breaking the original structure for the $\mathrm{SO}(5)$ WZW theory. However, such a term does not arise at least for the staggered flux phase of the $\mathrm{SU}(2)$ slave-boson theory since spinons are still at half filling even away from half filling. This is the reason why the $\mathrm{SU}(2)$ slave-boson framework is utilized in the present study. The other is how to construct couplings between doped holes and spin fluctuations. The second problem is our key issue.

We find that it is not easy to derive such couplings if we resort to the nonlinear $\sigma$ model description for the holon sector, derived in the previous study. [14] In the present paper we perform fermionization for the holon sector via flux attachment, where a Dirac theory with a chemical potential term due to the presence of finite density of holons is found in the staggered flux ansatz. Then, we can construct couplings between spin fluctuations and holons in the same way as the spinon sector since its theoretical structure is basically the same as that of the spinon part. Performing the standard gradient expansion, we have an effective field theory for $\mathrm{SO}(5)$ spin fluctuations, fermionic holons and their couplings. As a result, dynamics of doped holes in the antiferromagnetically correlated spin background turns out to be described by the $\mathrm{SO}(5)$ WZW theory for spin fluctuations, non-relativistic $\mathrm{QED}_{3}$ around Dirac nodes for doped holes, and interactions between valance bond fluctuations and holes.

Fermionic holons remind us of the slave-fermion representation. We compare our effective field theory with the $\mathrm{U}(1)$ slave-fermion theory of the t-J model, and discuss similarities and differences between them. Then, we solve the effective field theory in the Eliashberg framework, where momentum dependence in the fermion self-energy and vertex corrections are neglected, justified by Migdal theorem and large $N$ approximation with the fermion flavor number $N$ at least for the second assumption. 15. The Eliashberg framework allows us to construct LuttingerWard functional of its closed form, thus making it possible to solve the field theory self-consistently. Based on this framework, we discuss its phase diagram, thermodynamics, and transport near and away from the quantum critical point of our effective field theory, where non- 
Fermi liquid physics is found, analogous with that of the slave-fermion framework proposed recently. [16, 17]

An important feature of our field theory is the coupling term between valance bond fluctuations and doped holes. Considering that valance bond fluctuations are deeply related with monopole excitations of staggered $\mathrm{U}(1)$ gauge fields in the bosonic field theory for spin fluctuations, [18] the presence of such a coupling term allows us to investigate how dynamics of doped holes affects deconfinement of bosonic spinons. Remarkably, we find that such couplings help spin fractionalization near the quantum critical point of the $\mathrm{SO}(5)$ WZW theory.

We also apply our field theory to one dimensional antiferromagnetic doped Mott insulator, where spin fluctuations are described by the $\mathrm{SO}(4)$ WZW theory while charge excitations are represented by relativistic $\mathrm{QED}_{2}$. Since one dimensional study is well known, possible conclusions of our field theory in one dimension can justify validity of our description. Furthermore, reliable analytic techniques can be utilized in one dimension, thus this study would suggest an important feature how doped holes affect spinon deconfinement.

Finally, we discuss instability of the non-Fermi liquid metal phase against superconductivity and density waves of doped holes. As will be seen explicitly in the effective field theory [Eq. (10)], interactions between doped holes turn out to be mediated by both gauge and valance bond fluctuations. As a result, emergence of superconductivity from the non-Fermi liquid state is determined by competition between gauge and valance bond interactions. For particle-hole channel instabilities, we discuss that the homogeneous metallic phase can be stabilized at least when the flavor number of fermions (doped holes) is sufficiently large.

\section{EFFECTIVE FIELD THEORY}

Dynamics of doped holes in the antiferromagnetically correlated spin background is described by the t-J Hamiltonian

$$
H=-t \sum_{\langle i j\rangle}\left(c_{i \sigma}^{\dagger} c_{j \sigma}+H . c .\right)+J \sum_{\langle i j\rangle}\left(\vec{S}_{i} \cdot \vec{S}_{j}-\frac{1}{4} n_{i} n_{j}\right) .
$$

Introducing an $\mathrm{SU}(2)$ slave-boson representation for an electron field

$$
\begin{aligned}
& c_{i \uparrow}=\frac{1}{\sqrt{2}} h_{i}^{\dagger} \psi_{i+}=\frac{1}{\sqrt{2}}\left(b_{i 1}^{\dagger} f_{i 1}+b_{i 2}^{\dagger} f_{i 2}^{\dagger}\right), \\
& c_{i \downarrow}=\frac{1}{\sqrt{2}} h_{i}^{\dagger} \psi_{i-}=\frac{1}{\sqrt{2}}\left(b_{i 1}^{\dagger} f_{i 2}-b_{i 2}^{\dagger} f_{i 1}^{\dagger}\right),
\end{aligned}
$$

where $\psi_{i+}=\left(\begin{array}{c}f_{i 1} \\ f_{i 2}^{\dagger}\end{array}\right)$ and $\psi_{i-}=\left(\begin{array}{c}f_{i 2} \\ -f_{i 1}^{\dagger}\end{array}\right)$ are $\operatorname{SU}(2)$ spinon-spinors and $h_{i}=\left(\begin{array}{c}b_{i 1} \\ b_{i 2}\end{array}\right)$ is holon-spinor, one can rewrite the t-J model in terms of these fractionalized excitations with hopping and pairing fluctuations

$$
\begin{aligned}
& L=L_{0}+L_{s}+L_{h}, \quad L_{0}=J_{r} \sum_{\langle i j\rangle} \operatorname{tr}\left[U_{i j}^{\dagger} U_{i j}\right] \\
& L_{s}=\frac{1}{2} \sum_{i} \psi_{i \alpha}^{\dagger}\left(\partial_{\tau}-i a_{i 0}^{k} \tau_{k}\right) \psi_{i \alpha} \\
& +J_{r} \sum_{\langle i j\rangle}\left(\psi_{i \alpha}^{\dagger} U_{i j} \psi_{j \alpha}+\text { H.c. }\right), \\
& L_{h}=\sum_{i} h_{i}^{\dagger}\left(\partial_{\tau}-\mu-i a_{i 0}^{k} \tau_{k}\right) h_{i} \\
& +t_{r} \sum_{\langle i j\rangle}\left(h_{i}^{\dagger} U_{i j} h_{j}+\text { H.c. }\right),
\end{aligned}
$$

where the $\mathrm{SU}(2)$ matrix field is $U_{i j}=\left(\begin{array}{cc}-\chi_{i j}^{\dagger} & \eta_{i j} \\ \eta_{i j}^{\dagger} & \chi_{i j}\end{array}\right)$, and $J_{r}=\frac{3 J}{16}$ and $t_{r}=\frac{t}{2}$ are redefined couplings. [3] Since this decomposition representation enlarges the original electron Hilbert space, constraints are introduced via Lagrange multiplier fields $a_{i 0}^{k}$ with $k=1,2,3$.

In the $\mathrm{SU}(2)$ formulation Wen and Lee choose the staggered flux gauge [3]

$$
U_{i j}^{S F}=-\sqrt{\chi^{2}+\eta^{2}} \tau_{3} \exp \left[i(-1)^{i_{x}+i_{y}} \Phi \tau_{3}\right]
$$

with a phase $\Phi=\tan ^{-1}\left(\frac{\eta}{\chi}\right)$. Although the staggered flux ansatz breaks translational invariance, this formal symmetry breaking is restored via $\mathrm{SU}(2)$ fluctuations between nearly degenerate U(1) mean-field states. For example, one possible $\mathrm{U}(1)$ ground state, the d-wave pairing one $U_{i j}^{d S C}=-\chi \tau_{3}+(-1)^{i_{y}+j_{y}} \eta \tau_{1}$ can result from the $\mathrm{SU}(2)$ rotation $U_{i j}^{d S C}=W_{i} U_{i j}^{S F} W_{j}^{\dagger}$ with an $\mathrm{SU}(2)$ matrix $W_{i}=\exp \left\{i(-1)^{i_{x}+i_{y}} \frac{\pi}{4} \tau_{1}\right\}$. Then, our starting point becomes the following effective Lagrangian

$$
\begin{aligned}
& L_{S F}=\frac{1}{2} \sum_{i} \psi_{i \alpha}^{\dagger}\left(\partial_{\tau}-i a_{i 0}^{3} \tau_{3}\right) \psi_{i \alpha} \\
& +J_{r} \sum_{\langle i j\rangle}\left(\psi_{i \alpha}^{\dagger} U_{i j}^{S F} e^{i a_{i j}^{3} \tau_{3}} \psi_{j \alpha}+H . c .\right) \\
& +\sum_{i} h_{i}^{\dagger}\left(\partial_{\tau}-\mu-i a_{i 0}^{3} \tau_{3}\right) h_{i} \\
& +t_{r} \sum_{\langle i j\rangle}\left(h_{i}^{\dagger} U_{i j}^{S F} e^{i a_{i j}^{3} \tau_{3}} h_{j}+H . c .\right) \\
& +J_{r} \sum_{\langle i j\rangle} \operatorname{tr}\left[U_{i j}^{S F \dagger} U_{i j}^{S F}\right],
\end{aligned}
$$

where we have introduced only one kind of gauge field $a_{\mu}^{3}$ as important low energy fluctuations since other two ones, $a_{\mu}^{1}$ and $a_{\mu}^{2}$ are gapped due to Anderson-Higgs mechanism in the staggered flux phase. 14]

Our idea is to fermionize the holon sector attaching a 
fictitious flux to a holon field

$$
\begin{aligned}
& L_{S F}^{h}=\sum_{i} \eta_{i}^{\dagger}\left(\partial_{\tau}-\mu-i a_{i 0}^{3} \tau_{3}\right) \eta_{i} \\
& +t_{r} \sum_{\langle i j\rangle}\left(\eta_{i}^{\dagger} U_{i j}^{S F} e^{i a_{i j}^{3} \tau_{3}} e^{i c_{i j} \tau_{3}} \eta_{j}+H . c .\right) \\
& -i \sum_{i} c_{i 0}\left(\eta_{i}^{\dagger} \tau_{3} \eta_{i}-\frac{1}{2 \Theta}\left(\partial_{x} c_{y}-\partial_{y} c_{x}\right)_{i}\right),
\end{aligned}
$$

where a bosonic field variable $h_{i}$ now becomes a fermionic one $\eta_{i}=\left(\begin{array}{l}\eta_{i 1} \\ \eta_{i 2}\end{array}\right)$ with $\Theta=\pi$. It is important to notice that our flux attachment is performed in an opposite way for each isospin sector, confirmed by the presence of $\tau_{3}$ in $2 \Theta\left(\eta_{i}^{\dagger} \tau_{3} \eta_{i}\right)=\partial_{x} c_{y}-\partial_{y} c_{x}$. As a result, there is no net flux in the mean-field approximation of this construction, considering that the density of $b_{i 1}$ bosons is the same as that of $b_{i 2}$ bosons in the staggered flux phase. [14]

This observation is interesting since it suggests a connection with an $\mathrm{SU}(2)$ slave-fermion representation. If $a_{i j}^{3}$ is shifted to $a_{i j}^{3}-c_{i j}$, the Chern-Simons flux is transferred to spinons, turning their statistics into bosons. Then, we have a bosonic spinon description with a fermionic holon, nothing but the slave-fermion representation.

Performing the continuum approximation for the long wave length and low energy limits, we find an effective field theory in terms of only fermionic variables 14

$$
\begin{aligned}
& \mathcal{L}=\bar{\psi} \gamma_{\mu}\left(\partial_{\mu}-i a_{\mu}^{3} \tau_{3}\right) \psi+\frac{1}{2 e^{2}}\left(\epsilon_{\mu \nu \gamma} \partial_{\nu} a_{\gamma}^{3}\right)^{2} \\
& +\bar{\eta} \gamma_{\mu}\left(\partial_{\mu}-i a_{\mu}^{3} \tau_{3}-i c_{\mu} \tau_{3}\right) \eta-\mu_{h} \bar{\eta} \gamma_{0} \eta \\
& +\frac{i}{4 \Theta} c_{\mu} \epsilon_{\mu \nu \lambda} \partial_{\nu} c_{\lambda}
\end{aligned}
$$

Dirac structure [10] results from the staggered flux ansatz, where both $\psi$ and $\eta$ are 8 component spinors and Dirac gamma matrices are $\gamma_{0}=\left(\begin{array}{cc}\sigma_{3} & 0 \\ 0 & -\sigma_{3}\end{array}\right), \gamma_{1}=$ $\left(\begin{array}{cc}\sigma_{1} & 0 \\ 0 & -\sigma_{1}\end{array}\right)$, and $\gamma_{2}=\left(\begin{array}{cc}\sigma_{2} & 0 \\ 0 & -\sigma_{2}\end{array}\right) \cdot a_{\mu}^{3}$ is slave-boson $\mathrm{U}(1)$ gauge field, where a finite bare gauge charge $e$ is introduced. $c_{\mu}$ is Chern-Simons gauge field with its statistical angle $\Theta=\pi$. It is important to understand that spinons are still at half filling even away from half filling in the $\mathrm{SU}(2)$ formulation. The singleoccupancy constraint in the $\mathrm{SU}(2)$ representation is given by $f_{i 1}^{\dagger} f_{i 1}+f_{i 2}^{\dagger} f_{i 2}+b_{i 1}^{\dagger} b_{i 1}-b_{i 2}^{\dagger} b_{i 2}=1$. Thus, if the condition of $\left\langle b_{i 1}^{\dagger} b_{i 1}\right\rangle=\left\langle b_{i 2}^{\dagger} b_{i 2}\right\rangle=\frac{\delta}{2}$ with hole concentration $\delta$ is satisfied, we see $\left\langle f_{i 1}^{\dagger} f_{i 1}+f_{i 2}^{\dagger} f_{i 2}\right\rangle=1$, i.e., spinons are at half filling. As a result, a chemical potential term does not arise in the spinon sector. Actually, this was demonstrated for the staggered flux phase in the meanfield analysis of the $\mathrm{SU}(2)$ slave-boson theory. [14] On the other hand, a chemical potential term appears in the holon sector to form a Fermi pocket around the Dirac node.
To find an effective field theory for low energy spin fluctuations, it is necessary to consider physical symmetry of the spinon sector. We know that this symmetry is closely connected with both spin and Dirac spaces. Since spin $\mathrm{SU}(2)$ symmetry is hidden in the present 8 component representation, we consider the redundant representation $\Psi=\left(\begin{array}{c}\psi \\ \hat{\psi}\end{array}\right)$ of 16 components with a Dirac spinor $\hat{\psi} \equiv$ $i \tau_{2} \psi^{*}=\left(\begin{array}{c}f_{\downarrow} \\ -f_{\uparrow}^{\dagger}\end{array}\right)$, following Ref. [10]. Noting that the group space is composed of $G=G_{\text {Dirac }} \otimes G_{\text {gauge }} \otimes G_{\text {spin }}$, we see 15 generators associated with SU(4) symmetry [10] given by $I \otimes I \otimes \vec{\sigma}, \gamma_{3} \otimes I \otimes \vec{\sigma}, \gamma_{5} \otimes I \otimes \vec{\sigma}, i \gamma_{3} \gamma_{5} \otimes I \otimes I$, $\gamma_{3} \otimes \tau_{3} \otimes I, \gamma_{5} \otimes \tau_{3} \otimes I$, and $i \gamma_{3} \gamma_{5} \otimes \tau_{3} \otimes \vec{\sigma}$, where $\gamma_{3}=\left(\begin{array}{ll}0 & I \\ I & 0\end{array}\right)$ and $\gamma_{5}=\gamma_{0} \gamma_{1} \gamma_{2} \gamma_{3}=i\left(\begin{array}{cc}0 & I \\ -I & 0\end{array}\right)$ satisfying $\left[\gamma_{\mu}, \gamma_{3(5)}\right]_{+}=0$. This implies that symmetry equivalent operators via $\mathrm{SU}(4)$ have the same strength for instability, i.e., the same critical exponent for each correlation function. Actually, this was intensively discussed in Refs. [9, 10].

Recently, C. Xu and S. Sachdev have claimed existence of a novel spin liquid fixed point, where such an $\mathrm{SU}(4)$ symmetry is broken down to $\mathrm{SO}(5)$. 19] At this fixed point most relevant spin fluctuations are Neel vector and valance bond fluctuations, and they compete with each other since 10 generators of $I \otimes I \otimes \vec{\sigma}, \gamma_{3} \otimes I \otimes \vec{\sigma}, \gamma_{5} \otimes I \otimes \vec{\sigma}$, and $i \gamma_{3} \gamma_{5} \otimes I \otimes I$ remain and such spin fluctuations are symmetry equivalent operators via chiral rotation.

In this paper we also focus on competition between Neel and valance-bond fluctuations. Following Tanaka and $\mathrm{Hu}$, 11] we introduce an $\mathrm{SO}(5)$ superspin vector $\vec{v}=$ $\left(v_{1}, v_{2}, v_{3}, v_{4}, v_{5}\right)$, where the former three components form Neel vectors and the latter two ones represent $x$ and $y$ valance bond fluctuations, and consider the fermion mass term $-m \bar{\Psi}(\vec{v} \cdot \vec{\Gamma}) \Psi$ with $\vec{\Gamma}=\left(\sigma_{x}, \sigma_{y}, \sigma_{z}, i \gamma_{3}, i \gamma_{5}\right)$ for the spinon sector. On the other hand, the holon mass term becomes $-m_{\eta} \bar{\eta}\left(i \gamma_{3} v_{4}+i \gamma_{5} v_{5}\right)$ since it does not have spin. As a result, we find the following Lagrangian for symmetry "breaking"

$$
\begin{aligned}
& \mathcal{L}=\bar{\Psi} \gamma_{\mu}\left(\partial_{\mu}-i a_{\mu}^{3} \tau_{3}\right) \Psi-m \bar{\Psi}(\vec{v} \cdot \vec{\Gamma}) \Psi+\frac{1}{2 e^{2}}\left(\epsilon_{\mu \nu \gamma} \partial_{\nu} a_{\gamma}^{3}\right)^{2} \\
& +\bar{\eta} \gamma_{\mu}\left(\partial_{\mu}-i a_{\mu}^{3} \tau_{3}-i c_{\mu} \tau_{3}\right) \eta-\mu_{h} \bar{\eta} \gamma_{0} \eta+\frac{i}{4 \Theta} c_{\mu} \epsilon_{\mu \nu \lambda} \partial_{\nu} c_{\lambda} \\
& -m_{\eta} \bar{\eta}\left(i \gamma_{3} v_{4}+i \gamma_{5} v_{5}\right) \eta
\end{aligned}
$$

The next task is to perform integration of Dirac spinons and expand the resulting logarithmic action for the superspin vector. Based on the gradient expansion method, Tanaka and $\mathrm{Hu}$ have derived an $\mathrm{SO}(5)$ nonlinear $\sigma$ model with a WZW term ignoring gauge fluctuations, [1] $S_{s p i n}=S_{N L s M}+S_{W Z W}$, where $S_{N L s M}=\int d^{3} x \frac{1}{2 g}\left(\partial_{\mu} v_{k}\right)^{2}$ and $S_{W Z W}=$ $i \frac{2 \pi}{\text { Area }\left(S^{4}\right)} \int_{0}^{1} d t \int d^{3} x \epsilon_{a b c d e} v_{a} \partial_{t} v_{b} \partial_{\tau} v_{c} \partial_{x} v_{d} \partial_{y} v_{e} \quad$ with Area $\left(S^{4}\right)=\frac{2 \pi^{5 / 2}}{\Gamma(5 / 2)}$. Since Dirac fermions are massive in the symmetry "broken" phase and their fluctuations 
are ignored in the low energy limit, gauge-fluctuation corrections will be irrelevant considering that they can appear through fermion bubbles. Only one point that should be careful is a topological contribution, associated with an imaginary term. Although the WZW term is nicely derived in the absence of gauge fluctuations, an additional imaginary term may arise, a coupling term between a topologically nontrivial fermionic current and gauge field. [20] If we represent the Dirac spinor as $\psi_{n}=\left(\begin{array}{l}\chi_{n}^{+} \\ \chi_{n}^{-}\end{array}\right)$, where $\chi_{n}^{ \pm}$is a 2 component spinor with an isospin index $n=1,2$, one can see that each sector in the Dirac space gives rise to such a term. However, their signs are opposite, thus such terms are cancelled. [21] This is well known to be cancellation of parity anomaly in condensed matter physics. Another way to say this is that the signs of mass terms for Dirac fermions $\left(\chi_{n}^{+}\right.$and $\left.\chi_{n}^{-}\right)$are opposite, resulting in cancellation of the parity anomaly.

Based on the above discussion, we reach an effective field theory

$$
\begin{aligned}
& S=\int d^{3} x\left[\frac{1}{2 g}\left(\partial_{\mu} v_{k}\right)^{2}-m_{\eta} \bar{\eta}\left(i \gamma_{3} v_{4}+i \gamma_{5} v_{5}\right) \eta\right]+S_{W Z W} \\
& +\int d^{3} x\left[\bar{\eta} \gamma_{\mu}\left(\partial_{\mu}-i a_{\mu}^{3} \tau_{3}-i c_{\mu} \tau_{3}-i A_{\mu}\right) \eta-\mu_{h} \bar{\eta} \gamma_{0} \eta\right. \\
& \left.+\frac{i}{4 \Theta} c_{\mu} \epsilon_{\mu \nu \lambda} \partial_{\nu} c_{\lambda}+\frac{1}{2 e^{2}}\left(\epsilon_{\mu \nu \gamma} \partial_{\nu} a_{\gamma}^{3}\right)^{2}\right]
\end{aligned}
$$

where spin fluctuations are described by the SO(5) WZW theory. An interesting observation is that the ChernSimons contribution becomes irrelevant if the holon dynamics is in a critical phase. Shifting the slave-boson gauge field as $a_{\mu}^{3}-c_{\mu}$ and performing integration of Chern-Simons gauge fields, we obtain $\sim\left(\partial \times \partial \times a^{3}\right)$. $\left(\partial \times a^{3}\right)$. This contribution is irrelevant since it has a high scaling dimension owing to the presence of an additional derivative. Considering that the density of holons is finite to allow a Fermi surface (pocket around the Dirac point), it is natural to assume that the fermion sector is in criticality. We note that this kind of argument was well utilized previously. 222] As a result, we find an effective field theory for an antiferromagnetic doped Mott insulator problem

$$
\begin{aligned}
& S=\int d^{3} x\left[\frac{1}{2 g}\left(\partial_{\mu} v_{k}\right)^{2}-m_{\eta} \bar{\eta}\left(i \gamma_{3} v_{4}+i \gamma_{5} v_{5}\right) \eta\right] \\
& +\int d^{3} x\left[\bar{\eta} \gamma_{\mu}\left(\partial_{\mu}-i a_{\mu}^{3} \tau_{3}-i A_{\mu}\right) \eta-\mu_{h} \bar{\eta} \gamma_{0} \eta\right. \\
& \left.+\frac{1}{2 e^{2}}\left(\epsilon_{\mu \nu \gamma} \partial_{\nu} a_{\gamma}^{3}\right)^{2}\right]+S_{W Z W} .
\end{aligned}
$$

Several remarks are in order. First, the spin sector is described by the $\mathrm{SO}(5) \mathrm{WZW}$ theory even away from half filling, starting from the $\mathrm{SU}(2)$ slave-boson theory in the staggered flux gauge. Validity of this description will be further supported, comparing the present effective theory with the slave-fermion framework and applying it to one dimension. Second, dynamics of doped holes is described by $\mathrm{U}(1)$ gauge theory with finite density of fermionic holons around four Dirac nodes. Thus, non-Fermi liquid physics is expected naturally. Third, interactions between spin fluctuations and doped holes emerge as couplings between valance bond fluctuations and fermionic holons. Considering that valance bond fluctuations are deeply connected with monopole excitations of $\mathrm{CP}^{1}$ or staggered U(1) gauge fields in the bosonic field theory for spin fluctuations, this coupling form implies how dynamics of doped holes affects spin fractionalization, i.e., deconfinement of bosonic spinons. This will be discussed more deeply.

\section{CONNECTION WITH U(1) SLAVE-FERMION THEORY}

Although the effective field theory Eq. (10) has the similar spirit with the slave-fermion framework, it has an important different point. To understand this more clearly, we consider the $\mathrm{U}(1)$ slave-fermion representation

$$
c_{i \sigma}=\psi_{i}^{\dagger} b_{i \sigma}
$$

where $\psi_{i}$ and $b_{i \sigma}$ are fermionic holon and bosonic spinon, respectively. Inserting this representation into Eq. (1), we obtain the following expression for both exchangeinteraction and electron-hopping terms,

$$
\begin{aligned}
& J \sum_{i j}\left(\vec{S}_{i} \cdot \vec{S}_{j}-\frac{1}{4} n_{i} n_{j}\right) \\
& \rightarrow \frac{J}{2} \sum_{i j}\left|\Delta_{i j}^{b}\right|^{2}-J \sum_{i j}\left(\Delta_{i j}^{b \dagger} \epsilon_{\alpha \beta} b_{i \alpha} b_{j \beta}+H . c .\right), \\
& -t \sum_{i j}\left(c_{i \sigma}^{\dagger} c_{j \sigma}+H . c .\right) \rightarrow t \sum_{i j}\left(\chi_{j i}^{\psi} \chi_{i j}^{b}+H . c .\right) \\
& -t \sum_{i j}\left(b_{i \sigma}^{\dagger} \chi_{i j}^{b} b_{j \sigma}+H . c .\right)+t \sum_{i j}\left(\psi_{j}^{\dagger} \chi_{j i}^{\psi} \psi_{i}+H . c .\right),
\end{aligned}
$$

where each composite field is given by

$\Delta_{i j}=\sum_{\alpha \beta} \epsilon_{\alpha \beta} b_{i \alpha} b_{j \beta}, \quad \chi_{j i}^{\psi}=\sum_{\sigma} b_{i \sigma}^{\dagger} b_{j \sigma}, \quad \chi_{i j}^{b}=\psi_{i} \psi_{j}^{\dagger}$,

representing short-range antiferromagnetic correlations, ferromagnetic spin fluctuations, and hopping of holons, respectively.

In the low energy and long wave-length limits one can set the above collective fields as

$$
\Delta_{i j}=\Delta e^{i c_{i j}}, \quad \chi_{i j}^{b}=\chi_{b} e^{i a_{i j}}, \quad \chi_{i j}^{\psi}=\chi_{\psi} e^{i a_{i j}}
$$

where amplitude fluctuations are frozen to be their saddle-point values, and only phase fluctuations are 
kept importantly. Then, the resulting slave-fermion Lagrangian becomes

$$
\begin{aligned}
& L_{e f f}=L_{s}+L_{h}+L_{c}+L_{0}, \\
& L_{s}=\sum_{i} b_{i \sigma}^{\dagger}\left(\partial_{\tau}-\mu\right) b_{i \sigma}-t \chi_{b} \sum_{i j}\left(b_{i \sigma}^{\dagger} e^{i a_{i j}} b_{j \sigma}+H . c .\right) \\
& -J \Delta \sum_{i j}\left(e^{-i c_{i j}} \epsilon_{\alpha \beta} b_{i \alpha} b_{j \beta}+H . c .\right), \\
& L_{h}=\sum_{i} \psi_{i}^{\dagger} \partial_{\tau} \psi_{i}+t \chi_{\psi} \sum_{i j}\left(\psi_{j}^{\dagger} e^{-i a_{i j}} \psi_{i}+H . c .\right), \\
& L_{c}=i \sum_{i} \lambda_{i}\left(b_{i \sigma}^{\dagger} b_{i \sigma}+\psi_{i}^{\dagger} \psi_{i}-2 S\right), \\
& L_{0}=N_{L}\left(J \Delta^{2}+4 t \chi_{\psi} \chi_{b}\right),
\end{aligned}
$$

where $\lambda_{i}$ in $L_{c}$ is a Lagrange multiplier field to impose the single occupancy constraint with $S=1 / 2$, and $N_{L}$ in $L_{0}$ is number of lattice sites.

Comparing Eq. (10) with Eq. (14), one will find several different points. First, the spectrum of holon excitations is not relativistic in Eq. (14). However, this can be adjusted, allowing $\pi$ flux in the U(1) gauge field $a_{i j}$, i.e., $\sum_{\square} a_{i j}=\pi$. Then, four Dirac nodes arise, and hole doping gives finite density of holons, resulting in the hole pockets around the nodes.

Second, spin dynamics is described by usual spin 1 excitations in Eq. (10) while it is expressed with spin $1 / 2$ fractionalized spinons in Eq. (14). However, the presence of the WZW term in Eq. (10) may give rise to spin fractionalization as one dimensional physics [12] or the previous proposal [6] for deconfined quantum criticality. If we start from Eq. (14), we focus on two kinds of gauge fluctuations, corresponding to staggered $\mathrm{U}(1)$ gauge fields $c_{i j}$ and uniform $\mathrm{U}(1)$ gauge fields $a_{i j}$, respectively. In particular, the staggered $\mathrm{U}(1)$ gauge field $c_{i j}$ turns out to be the same as the $\mathrm{CP}^{1}$ gauge field if the Schwinger-boson effective Lagrangian is mapped onto the $\mathrm{CP}^{1}$ gauge theory of the $\mathrm{O}(3)$ nonlinear $\sigma$ model in the long wave-length limit. [18 In this respect, if we assume that staggered gauge fluctuations mediate confining interactions between spinons, we expect to see spin 1 fluctuations described by a $\sigma$ model-type theory. However, the fate of valance bond fluctuations is not clear in this case.

Third, the coupling structure between spin fluctuations and doped holes in Eq. (10) differs from that in Eq. (14). In the slave-fermion Lagrangian Eq. (14) direct couplings between spin and charge degrees of freedom do not exist although nonlocal current-current interactions appear in the low energy limit, associated with the single occupancy constraint. Even if we assume staggered gauge fluctuations confining, it is difficult to find a coupling term between spin fluctuations and doped holes. On the other hand, our effective field theory Eq. (10) exhibits its direct coupling term explicitly. This coupling term is an important ingredient for dynamics of doped holes in the quantum antiferromagnet. As demonstrated intensively in the previous work, [18] valance bond fluctuations are deeply related with instanton (monopole) excitations of staggered $\mathrm{U}(1)$ gauge fields $c_{i j}$. Actually, $v_{4}$ and $v_{5}$ fields can be identified with an instanton operator in the presence of a topological $\theta$ term. This implies that the presence of doped holes affects spinon confinement directly via their couplings. In the cumulant expansion for the coupling term one can see that holon dynamics modifies monopole-monopole correlations, giving rise to dissipation. As a result, an effective theory for monopole excitations away from half filling is changed seriously from that at half filling. In the next section we will see that the presence of doped holes helps bosonic spinons deconfined near the quantum critical point.

\section{PHYSICAL PROPERTIES}

\section{A. Eliashberg framework}

Three kinds of field variables, that is, fermionic holon, $\mathrm{SO}$ (5) superspin vector, and uniform $\mathrm{U}(1)$ gauge field make our effective field theory complicated. In this respect it is not easy to treat such all degrees of freedom self-consistently. Recently, it was explicitly demonstrated that Eliashberg framework is the minimal self-consistent treatment for an effective field theory near its quantum critical point. 15. The Eliashberg treatment neglects momentum dependence of a fermion self-energy and vertex corrections. The first assumption is based on the fact that momentum dependence of a fermion self-energy is regular, and singular physics arises from its frequency dependence. This can be checked explicitly at least in the one loop level. The second assumption is more serious than the first one, sometimes called Migdal theorem. 23] When fermions are much faster than bosons, vertex corrections can be neglected since the pre-factor in the renormalized vertex is given by the ratio of fermion and boson velocities. However, this turns out to be not sufficient for the Eliashberg framework. [15] Another parameter is shown to be need, that is, the fermion flavor number $N$. In the large $N$ limit the Eliashberg framework is justified.

For the Eliashberg treatment we rewrite the effective field theory as follows

$$
\begin{aligned}
& S=S_{\eta}+S_{v}+S_{a}+S_{i n t}, \\
& S_{\eta}=\int d^{3} x\left\{\bar{\eta} \gamma_{\mu}\left(\partial_{\mu}-i A_{\mu}\right) \eta-\mu_{h} \bar{\eta} \gamma_{0} \eta\right\}, \\
& S_{v}=\int d^{3} x\left\{\frac{1}{2 g}\left(\partial_{\mu} v_{k}\right)^{2}+m_{v}^{2}\left(\left|v_{k}\right|^{2}-1\right)\right\}+S_{W Z W}, \\
& S_{a}=\int d^{3} x\left\{\frac{1}{2 e^{2}}\left(\epsilon_{\mu \nu \gamma} \partial_{\nu} a_{\gamma}^{3}\right)^{2}\right\}, \\
& S_{i n t}=\int d^{3} x\left\{-m_{\eta} \bar{\eta}\left(i \gamma_{3} v_{4}+i \gamma_{5} v_{5}\right) \eta-i a_{\mu}^{3} \bar{\eta} \gamma_{\mu} \tau_{3} \eta\right\},
\end{aligned}
$$

where $m_{v}^{2}$ represents mass of superspin vector bosons arising from the $\mathrm{SO}(5)$ rotor constraint $\sum_{k=1}^{5}\left|v_{k}\right|^{2}=1$. 
It will be determined self-consistently.

Performing the cumulant expansion for $S_{i n t}$, we find an effective action $S_{\text {eff }}=S_{v}+S_{\eta}+S_{a}-\frac{1}{2}\left(\left\langle S_{\text {int }}^{2}\right\rangle-\left\langle S_{\text {int }}\right\rangle^{2}\right)$. Although this expression is for the second order, it includes an infinite order actually. Accordingly, we can construct the corresponding Luttinger-Ward functional in the Eliashberg framework

$$
\begin{aligned}
& F_{L W}=F_{L W}^{\eta}+F_{L W}^{v}+F_{L W}^{a}+Y_{v}+Y_{a}, \\
& F_{L W}^{\eta}=-T \sum_{i \omega} \int \frac{d^{d} k}{(2 \pi)^{d}} \operatorname{tr}\left[\ln \left\{g_{\eta}^{-1}(k, i \omega)+\Sigma_{\eta}(i \omega)\right\}\right. \\
& \left.-\Sigma_{\eta}(i \omega) G_{\eta}(k, i \omega)\right], \\
& F_{L W}^{v}=T \sum_{i \Omega} \int \frac{d^{d} q}{(2 \pi)^{d}}\left[\sum _ { m , n = 1 } ^ { 5 } \operatorname { l n } \left\{d_{v}^{-1}(q, i \Omega) \delta_{m n}\right.\right. \\
& \left.\left.+\Pi_{v}^{m n}(q, i \Omega)\right\}-\sum_{m, n=1}^{5} \Pi_{v}^{m n}(q, i \Omega) D_{v}^{m n}(q, i \Omega)\right]-m_{v}^{2}, \\
& F_{L W}^{a}=T \sum_{i \Omega} \int \frac{d^{d} q}{(2 \pi)^{d}}\left[\ln \left\{d_{a}^{-1}(q, i \Omega)+\Pi_{a}(q, i \Omega)\right\}\right. \\
& \left.-\Pi_{a}(q, i \Omega) D_{a}(q, i \Omega)\right], \\
& Y_{v}=-\frac{m_{\eta}^{2}}{2} T \sum_{i \omega} \int \frac{d^{d} k}{(2 \pi)^{d}} T \sum_{i \Omega} \int \frac{d^{d} q}{(2 \pi)^{d}} \\
& \sum_{m, n=4}^{5} \operatorname{tr}\left[D_{v}^{m n}(q, i \Omega) \gamma_{m} G_{\eta}(k+q, i \omega+i \Omega) \gamma_{n} G_{\eta}(k, i \omega)\right], \\
& Y_{a}=-\frac{1}{2} T \sum_{i \omega} \int \frac{d^{d} k}{(2 \pi)^{d}} T \sum_{i \Omega} \int \frac{d^{d} q}{(2 \pi)^{d}} \\
& \operatorname{tr}\left[D_{a}^{\mu \nu}(q, i \Omega) \gamma_{\mu} \tau_{3} G_{\eta}(k+q, i \omega+i \Omega) \gamma_{\nu} \tau_{3} G_{\eta}(k, i \omega)\right](16)
\end{aligned}
$$

$G_{\eta}(k, i \omega)$ in $F_{L W}^{\eta}$ is a renormalized propagator for holons, given by $G_{\eta}(k, i \omega)=\left\{g_{\eta}^{-1}(k, i \omega)+\Sigma_{\eta}(i \omega)\right\}^{-1}$, where $g_{\eta}(k, i \omega)=\left(i \gamma_{0} \omega+i \gamma_{i} k_{i}+\mu_{h} \gamma_{0}\right)^{-1}$ is its bare propagator, and $\Sigma_{\eta}(i \omega)$ is its momentum-independent selfenergy. $D_{v}^{m n}(q, i \Omega)$ in $F_{L W}^{v}$ is a renormalized propagator for superspin vector fields, given by $D_{v}^{m n}(q, i \Omega)=$ $\left\{d_{v}^{-1}(q, i \Omega) \delta_{m n}+\Pi_{v}^{m n}(q, i \Omega)\right\}^{-1}$, where $d_{v}(q, i \Omega)=$ $\left(\frac{q^{2}+\Omega^{2}}{2 g}+m_{v}^{2}\right)^{-1}$ is its bare propagator, and $\Pi_{v}^{m n}(q, i \Omega)$ is its self-energy. $D_{a}(q, i \Omega)$ in $F_{L W}^{a}$ is a renormalized kernel for the gauge propagator $D_{a}^{\mu \nu}(q, i \Omega)=D_{a}(q, i \Omega)\left(\delta_{\mu \nu}-\right.$ $\left.\frac{q_{\mu} q_{\nu}}{q^{2}}\right)$, given by $D_{a}(q, i \Omega)=\left\{d_{a}^{-1}(q, i \Omega)+\Pi_{a}(q, i \Omega)\right\}^{-1}$, where $d_{a}(q, i \Omega)=\left(\frac{q^{2}+\Omega^{2}}{2 e^{2}}\right)^{-1}$ is its bare kernel, and $\Pi_{a}(q, i \Omega)$ is its self-energy in $\Pi_{a}^{\mu \nu}(q, i \Omega)=$ $\Pi_{a}(q, i \Omega)\left(\delta_{\mu \nu}-\frac{q_{\mu} q_{\nu}}{q^{2}}\right) . Y_{v}$ is introduced for self-energy corrections resulting from the first term in $S_{\text {int }}$ of Eq. (15) while $Y_{a}$ is for those arising from the second term of $S_{i n t}$. In the self-energy functional $Y_{v} \gamma_{4}$ should be replaced with $\gamma_{3}$, where this problem appears from our notation form.

It is important to notice that Luttinger-Ward functional is not usually written in a closed form. However, the Luttinger-Ward functional can be written in its closed form at least for the Eliashberg framework. 24] Actually, performing variation for the Luttinger-Ward functional with respect to each self-energy, i.e., $\frac{\delta F_{L W}}{\delta \Sigma_{\eta}(i \omega)}=0$, $\frac{\delta F_{L W}}{\delta \Pi_{v}^{m n}(q, i \Omega)}=0$, and $\frac{\delta F_{L W}}{\delta \Pi_{a}^{\mu \nu}(q, i \Omega)}=0$, we find self-consistent Eliashberg equations

$$
\begin{aligned}
& \Sigma_{\eta}(i \omega)=m_{\eta}^{2} T \sum_{i \Omega} \int \frac{d^{d} q}{(2 \pi)^{d}} \sum_{m, n=4}^{5} \\
& D_{v}^{m n}(q, i \Omega) \gamma_{m} G_{\eta}\left(k_{F}+q, i \omega+i \Omega\right) \gamma_{n}+T \sum_{i \Omega} \int \frac{d^{d} q}{(2 \pi)^{d}} \\
& D_{a}^{\mu \nu}(q, i \Omega) \gamma_{\mu} \tau_{3} G_{\eta}\left(k_{F}+q, i \omega+i \Omega\right) \gamma_{\nu} \tau_{3}, \\
& \Pi_{v}^{m n}(q, i \Omega)=T \sum_{i \omega} \int \frac{d^{d} k}{(2 \pi)^{d}} \sum_{m, n=4}^{5} \\
& \left(-\frac{m_{\eta}^{2}}{2} \operatorname{tr}\left[\gamma_{m} G_{\eta}(k+q, i \omega+i \Omega) \gamma_{n} G_{\eta}(k, i \omega)\right]\right), \\
& \Pi_{a}^{\mu \nu}(q, i \Omega)=T \sum_{i \omega} \int \frac{d^{d} k}{(2 \pi)^{d}} \\
& \left(-\frac{1}{2} \operatorname{tr}\left[\gamma_{\mu} \tau_{3} G_{\eta}(k+q, i \omega+i \Omega) \gamma_{\nu} \tau_{3} G_{\eta}(k, i \omega)\right]\right) .
\end{aligned}
$$

The holon self-energy results from both valance bond and gauge fluctuations, where $\gamma_{4} \rightarrow \gamma_{3}$ is performed. The superspin vector self-energy arises from holon fluctuations, where $\gamma_{4}$ is also replaced with $\gamma_{3}$. Notice $\Pi_{v}^{m n}(q, i \Omega)=0$ for $m, n=1,2,3$. The gauge field self-energy appears from holon current fluctuations. Eqs. (16) and (17) complete the Eliashberg framework.

\section{B. Simplification of Luttinger-Ward functional}

Using the Eliashberg equations (17), one can simplify the Luttinger-Ward functional Eq. (16) as follows

$$
\begin{aligned}
& F_{L W}=-T \sum_{i \omega} \int \frac{d^{d} k}{(2 \pi)^{d}} \operatorname{tr}\left[\ln \left\{g_{\eta}^{-1}(k, i \omega)+\Sigma_{\eta}(i \omega)\right\}\right. \\
& \left.-\Sigma_{\eta}(i \omega) G_{\eta}(k, i \omega)\right] \\
& +T \sum_{i \Omega} \int \frac{d^{d} q}{(2 \pi)^{d}}\left[\sum_{m, n=1}^{5} \ln \left\{d_{v}^{-1}(q, i \Omega) \delta_{m n}+\Pi_{v}^{m n}(q, i \Omega)\right\}\right] \\
& -m_{v}^{2}+T \sum_{i \Omega} \int \frac{d^{d} q}{(2 \pi)^{d}} \ln \left\{d_{a}^{-1}(q, i \Omega)+\Pi_{a}(q, i \Omega)\right\},
\end{aligned}
$$

where the self-energy parts for superspin vector and U(1) gauge fields are cancelled out from the use of Eq. (17). Then, one can see that the holon free energy is nothing 
but the free energy of Fermi liquid as follows

$$
\begin{aligned}
& F_{L W}^{\eta} \approx-N_{\eta} T \sum_{i \omega} \int \frac{d^{d} k}{(2 \pi)^{d}} \ln \left\{2 \mu_{h}(i \omega)+\mu_{h}^{2}-\omega^{2}-k^{2}\right\} \\
& \approx-N_{\eta} \rho_{\eta} T \sum_{i \omega}|\omega|=-\frac{\pi N_{\eta} \rho_{\eta}}{6} T^{2}=F_{F L}^{\eta}
\end{aligned}
$$

Here, $\rho_{\eta}$ is the density of states around the Dirac node, and the number of Dirac nodes is $N_{\eta}=4 . f(y)=\frac{1}{e^{y}+1}$ is the Fermi-Dirac distribution function with temperature scaling. As a result, we find the Eliashberg free energy of our effective field theory

$$
\begin{aligned}
& F_{L W}=-\frac{\pi N_{\eta} \rho_{\eta}}{6} T^{2}-m_{v}^{2} \\
& +T \sum_{i \Omega} \int \frac{d^{d} q}{(2 \pi)^{d}}\left[\sum_{m, n=1}^{5} \ln \left\{d_{v}^{-1}(q, i \Omega) \delta_{m n}+\Pi_{v}^{m n}(q, i \Omega)\right\}\right. \\
& +T \sum_{i \Omega} \int \frac{d^{d} q}{(2 \pi)^{d}} \ln \left\{d_{a}^{-1}(q, i \Omega)+\Pi_{a}(q, i \Omega)\right\}
\end{aligned}
$$

The remaining thing is to evaluate each selfenergy. The superspin vector self-energy $\Pi_{v}^{m n}(q, i \Omega)=$ $\Pi_{v}(q, i \Omega) \delta_{m n}$ for $m, n=4,5$ is found to be

$$
\Pi_{v}(q, i \Omega) \approx \frac{\pi N_{\eta} m_{\eta}^{2} \rho_{\eta}}{4} \frac{|\Omega|}{q} .
$$

This is nothing but the standard Landau damping term, originating from fermion excitations near the Fermi surface. Since the density of holons is finite due to hole doping, emergence of the Landau damping term is quite natural. Then, the renormalized propagator for superspin fluctuations is given by

$$
\begin{aligned}
& D_{v}^{m n}(q, i \Omega)=\frac{\delta_{m n}}{\frac{q^{2}+\Omega^{2}}{2 g}+m_{v}^{2}}, \quad \text { for } m, n=1,2,3, \\
& D_{v}^{m n}(q, i \Omega)=\frac{\delta_{m n}}{\frac{q^{2}+\Omega^{2}}{2 g}+m_{v}^{2}+\frac{\pi N_{\eta} m_{\eta}^{2} \rho_{\eta}}{4} \frac{|\Omega|}{q}} \\
& \approx \frac{\delta_{m n}}{\frac{q^{2}}{2 g}+m_{v}^{2}+\frac{\pi N_{\eta} m_{\eta}^{2} \rho_{\eta}}{4} \frac{|\Omega|}{q}}, \quad \text { for } m, n=4,5,(22)
\end{aligned}
$$

implying that antiferromagnetic spin fluctuations are described by $z=1$ theory while valance bond fluctuations are expressed by $z=3$ theory, where $z$ is the dynamical exponent. The gauge self-energy is also given by the Landau damping term $\Pi_{a}(q, i \Omega)=\frac{\pi N_{\eta} \rho_{\eta}}{4} \frac{|\Omega|}{q}$, thus the renormalized gauge propagator becomes

$$
D_{a}(q, i \Omega) \approx \frac{1}{\frac{q^{2}}{2 e^{2}}+\frac{\pi N_{\eta} \rho_{\eta}}{4} \frac{|\Omega|}{q}}
$$

described by $z=3$ critical theory.

Inserting bosonic self-energies into Eq. (20), we find the final expression for the Eliashberg free energy

$$
\begin{aligned}
& F_{L W}=-\frac{\pi N_{\eta} \rho_{\eta}}{6} T^{2}-\frac{\xi^{-2}}{2 g}+T \sum_{i \Omega} \int \frac{d^{d} q}{(2 \pi)^{d}} \\
& \left\{3 \ln \left(q^{2}+\Omega^{2}+\xi^{-2}\right)+2 \ln \left(q^{2}+\xi^{-2}+\gamma_{v} \frac{|\Omega|}{q}\right)\right\} \\
& +T \sum_{i \Omega} \int \frac{d^{d} q}{(2 \pi)^{d}} \ln \left(q^{2}+\gamma_{a} \frac{|\Omega|}{q}\right),
\end{aligned}
$$

where

$$
\xi^{-2}=2 g m_{v}^{2}, \quad \gamma_{v}=\frac{\pi g N_{\eta} m_{\eta}^{2} \rho_{\eta}}{2}, \quad \gamma_{a}=\frac{\pi e^{2} N_{\eta} \rho_{\eta}}{2}
$$

represent the correlation length for superspin fluctuations, Landau damping coefficient for superspin fields, and that for gauge fields.

Several remarks about the Eliashberg framework are in order. First, the Landau damping contribution does not depend on the fermion self-energy in the Eliashberg framework, allowing one to use a bare fermion propagator instead of its full green's function. 15] Second, $z=3$ criticality supports Migdal theorem since on-shell (or resonance) fermion momenta are larger than on-shell boson one at the same energy. Third, the number of Dirac nodes $N_{\eta}$ plays the same role as the fermion flavor number in the Eliashberg framework. In this respect the Eliashberg framework works well for our effective field theory.

\section{Phase diagram}

Performing variation of the free energy Eq. (24) with respect to the correlation length, i.e., $\frac{\partial F_{L W}}{\partial \xi^{-2}}=0$, we obtain the self-consistent equation for the correlation length in the Eliashberg framework

$$
\begin{aligned}
& 1=2 g T \sum_{i \Omega} \int \frac{d^{d} q}{(2 \pi)^{d}}\left(\frac{3}{q^{2}+\Omega^{2}+\xi^{-2}}\right. \\
& \left.+\frac{2}{q^{2}+\xi^{-2}+\gamma_{v} \frac{|\Omega|}{q}}\right) .
\end{aligned}
$$

Notice that interactions between valance bond fluctuations and holons result in the $z=3$ part.

Performing integration, we find the following expression for the correlation length

$$
\begin{aligned}
& 1=\frac{3 g}{\pi} T\left\{\ln \sinh \left(\frac{\Lambda}{2 T}\right)-\ln \sinh \left(\frac{\xi^{-1}}{2 T}\right)\right\} \\
& +\frac{4 g}{3 \pi} T \ln \left\{\sinh \left(\frac{\left(\xi^{3} \gamma_{v}\right)^{-1}}{2 T}\right)\right\} \\
& -2 T\left[-\frac{\left(\xi^{3} \gamma_{v}\right)^{-2}}{4 T}-\frac{\pi^{2}}{6} T-\left(\xi^{3} \gamma_{v}\right)^{-1} \ln \left(1-e^{-\frac{\left(\xi^{3} \gamma_{v}\right)^{-1}}{T}}\right)\right. \\
& \left.+\left(\xi^{3} \gamma_{v}\right)^{-1} \ln \left\{\sinh \left(\frac{\left(\xi^{3} \gamma_{v}\right)^{-1}}{2 T}\right)\right\}+T \sum_{k=1}^{\infty} \frac{e^{-k \frac{\left(\xi^{3} \gamma_{v}\right)^{-1}}{T}}}{k^{2}}\right] \\
& +\frac{2 g}{3} T\left[\ln \sinh \left(\frac{\Lambda}{2 T}\right)-\ln \sinh \left(\frac{\left(\xi^{3} \gamma_{v}\right)^{-1}}{2 T}\right)\right] .
\end{aligned}
$$




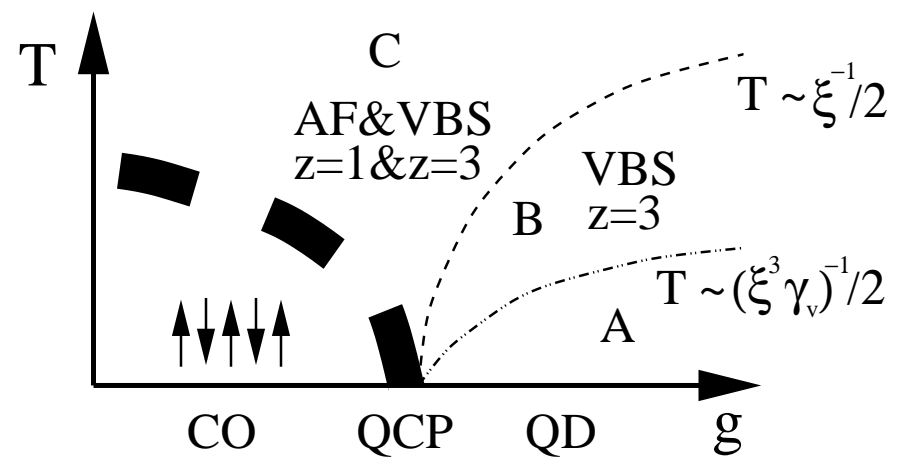

FIG. 1: (Color online) Schematic phase diagram : CO, QD, and QCP represent classically ordered, quantum disordered, and quantum critical point, respectively. Two crossover scales emerge as $T \sim \frac{\left(\xi^{3} \gamma_{v}\right)^{-1}}{2}$ and $T \sim \frac{\xi^{-1}}{2}$, identifying three regimes. In regime $(\mathrm{A})$ of $T<\frac{\left(\xi^{3} \gamma_{v}\right)^{-1}}{2}<\frac{\xi^{-1}}{2}$ both $z=1$ $\mathrm{AF}$ and $z=3 \mathrm{VBS}$ fluctuations are gapped while in regime (C) of $\frac{\left(\xi^{3} \gamma_{v}\right)^{-1}}{2}<\frac{\xi^{-1}}{2}<T$ both spin fluctuations are critical, that is, in the quantum critical regime. In regime (B) of $\frac{\left(\xi^{3} \gamma_{v}\right)^{-1}}{2}<T<\frac{\xi^{-1}}{2}$ only $z=3$ VBS fluctuations are critical, and $z=1 \mathrm{AF}$ ones are gapped.

As shown in this equation, there exist three regimes, (A) $T<\frac{\left(\xi^{3} \gamma_{v}\right)^{-1}}{2}<\frac{\xi^{-1}}{2}$, (B) $\frac{\left(\xi^{3} \gamma_{v}\right)^{-1}}{2}<T<\frac{\xi^{-1}}{2}$, and $(\mathrm{C})$ $\frac{\left(\xi^{3} \gamma_{v}\right)^{-1}}{2}<\frac{\xi^{-1}}{2}<T$, emerging from coexistence of $z=1$ (antiferromagnetic) and $z=3$ (valance bond) fluctuations. In regime (A) both $z=1$ and $z=3$ fluctuations are gapped while in regime $(\mathrm{C})$ both spin fluctuations are critical, that is, in the quantum critical regime. In regime (B) only valance bond fluctuations $(z=3)$ are critical, and $z=1$ antiferromagnetic ones are gapped. The phase diagram is shown in Fig. 1.

Considering $\frac{\left(\xi^{3} \gamma_{v}\right)^{-1}}{2} \ll \frac{\xi^{-1}}{2}$ near the quantum critical point, we can simplify the above equation, and find that antiferromagnetic fluctuations play an important role in determining the $\mathrm{SO}(5)$ superspin correlation length near the quantum critical point. As a result, we obtain the following expression for the correlation length

$$
\begin{aligned}
\xi^{-1}\left(g \sim g_{c} ; T<\frac{\xi^{-1}}{2}\right) & \approx\left\{\left(1+\frac{2 \pi}{9}\right) \Lambda-\frac{2 \pi}{3 g}\right\} \\
& \equiv \frac{2 \pi}{3}\left(\frac{1}{g_{c}}-\frac{1}{g}\right), \\
\xi^{-1}\left(g \sim g_{c} ; T>\frac{\xi^{-1}}{2}\right) & =2 T e^{\frac{\pi}{3} \frac{\left(\frac{1}{g_{c}}-\frac{1}{g}\right)}{T}},
\end{aligned}
$$

where $g_{c}=\left(\frac{3}{2 \pi}+\frac{1}{3}\right) \Lambda$ is the quantum critical point. 225] Recalling $g(\delta) \propto m^{2}(\delta)$ in the gradient expansion and $m^{2}(\delta) \propto\left|\delta-\delta_{0}\right|$ with hole concentration $\delta<\delta_{0}$ near the quantum critical point, we find the critical hole concentration in the Eliashberg framework

$$
\delta_{c}=\delta_{0}-\frac{1}{c}\left\{\left(\frac{3}{2 \pi}+\frac{1}{3}\right) \Lambda\right\}
$$

where $c$ is a positive numerical constant. Thus, an antiferromagnetically ordered phase appears in $\delta<\delta_{c}$, and a quantum disordered state arises in $\delta>\delta_{c}$. The nature of the disordered state will be determined by $\mathrm{SO}(5)$ symmetry breaking effective potentials.

\section{Deconfined quantum critical point}

We consider how interactions between valance bond fluctuations and holons affect the deconfined quantum critical point, proposed to exist at half filling. As discussed before, valance bond fluctuations can be identified with monopole excitations of staggered gauge fields. Introducing $\Psi=\frac{1}{\sqrt{2}}\left(v_{4}+i v_{5}\right)$ and considering $\mathrm{SO}(5)$ symmetry breaking for the WZW term as follows in Ref. [11], we find an effective field theory for monopole or valance bond fluctuations from the effective field theory Eq. (10),

$$
\begin{aligned}
& S_{V B}=T \sum_{i \Omega} \int \frac{d^{2} q}{(2 \pi)^{2}} \\
& \Psi^{\dagger}(q, i \Omega)\left(\frac{q^{2}}{2 g}+\frac{\pi N_{\eta} m_{\eta}^{2} \rho_{\eta}}{4} \frac{|\Omega|}{q}\right) \Psi(q, i \Omega) \\
& -\int_{0}^{\beta} d \tau \int d^{2} r y_{m}\left(\Psi^{4}+\Psi^{\dagger 4}\right) .
\end{aligned}
$$

Here, the cubic power in the last term results from the WZW term with $\mathrm{SO}(5)$ symmetry breaking, where $y_{m}$ is the monopole fugacity. If the topological $\theta$ term is not taken into account, the condensation-induced term will be given by $-\int_{0}^{\beta} d \tau \int d^{2} r y_{m}\left(\Psi+\Psi^{\dagger}\right)$.

An important point is that dynamics of valance bond excitations is described by $z=3$ critical theory at the quantum critical point. As a result, two spacial dimensions are already in the upper critical dimension, thus higher order interactions beyond the Gaussian term are irrelevant. It means that the WZW-induced cubic term can be neglected safely at the quantum critical point. Equivalently, the monopole fugacity vanishes at the quantum critical point, indicating deconfinement of bosonic spinons. We point out that the topological term plays an important role for deconfinement even away from half filling. If the topological term is ignored, the monopole-fugacity term of linear in $\Psi$ is relevant, giving rise to confinement.

Although proliferation of monopole excitations is prohibited due to fermion excitations, monopoles are in a critical state of $z=3$ instead of a gapped phase. Such critical valance bond fluctuations will affect holon dynamics. This feedback effect to holon dynamics is an important ingredient for the source of non-Fermi liquid physics near the quantum critical point. 


\section{E. Thermodynamics}

We study thermodynamics in each regime. We start from the following expression for the Eliashberg free energy

$$
\begin{aligned}
& F_{L W}=-\frac{\pi N_{\eta} \rho_{\eta}}{6} T^{2}-\frac{\xi^{-2}}{2 g} \\
& +\frac{3}{2 \pi} T \int_{0}^{\infty} d q q \ln \left\{2 \sinh \left(\frac{\sqrt{q^{2}+\xi^{-2}}}{2 T}\right)\right\} \\
& -\frac{1}{\pi^{2}} \int_{0}^{\infty} d q q \int_{0}^{\infty} d \nu \operatorname{coth}\left(\frac{\nu}{2 T}\right) \tan ^{-1}\left(\gamma_{v} \frac{\nu}{q\left[q^{2}+\xi^{-2}\right]}\right) \\
& -\frac{1}{2 \pi^{2}} \int_{0}^{\infty} d \nu \operatorname{coth}\left(\frac{\nu}{2 T}\right) \int_{0}^{\infty} d q q \tan ^{-1}\left(\gamma_{a} \frac{\nu}{q^{3}}\right) .
\end{aligned}
$$

The first term is due to the Fermi liquid contribution of holon excitations, and the second is the correlation length term. The third contribution results from $z=$ 1 antiferromagnetic fluctuations while the fourth term arises from $z=3$ valance bond excitations. The last term originates from $z=3$ gauge fluctuations.

Considering the specific heat coefficient given by $\gamma(T)=\frac{C(T)}{T}=-\frac{\partial^{2} F_{L W}(T)}{\partial T^{2}}$, we obtain its analytic expression in each regime,

$$
\begin{aligned}
& \gamma\left(T>\frac{\left(\gamma_{v} \xi^{3}\right)^{-1}}{2}\right) \approx 2^{8 / 3}\left(\frac{1}{2 \pi^{2}}+\frac{1}{8 \pi}\right)\left(2 \gamma_{v}^{2 / 3}+\gamma_{a}^{2 / 3}\right) \\
& {\left[\int_{0}^{\infty} d y\left\{-\frac{y^{5 / 3}}{\sinh ^{2} y}+\frac{y^{8 / 3} \operatorname{coth} y}{\sinh ^{2} y}\right\}\right] T^{-1 / 3}} \\
& \gamma\left(T<\frac{\left(\gamma_{v} \xi^{3}\right)^{-1}}{2}\right) \approx 2^{8 / 3}\left(\frac{1}{2 \pi^{2}}+\frac{1}{8 \pi}\right) \gamma_{a}^{2 / 3} \\
& {\left[\int_{0}^{\infty} d y\left\{-\frac{y^{5 / 3}}{\sinh ^{2} y}+\frac{y^{8 / 3} \operatorname{coth} y}{\sinh ^{2} y}\right\}\right] T^{-1 / 3}}
\end{aligned}
$$

where only dominant contributions are shown. As discussed previously, we have three regimes, (A) $T<$ $\frac{\left(\xi^{3} \gamma_{v}\right)^{-1}}{2}$ where both antiferromagnetic and valance bond fluctuations are gapped, (B) $\frac{\left(\xi^{3} \gamma_{v}\right)^{-1}}{2}<T<\frac{\xi^{-1}}{2}$ where only valance bond fluctuations are critical, and (C) $\frac{\xi^{-1}}{2}<$ $T$ where both antiferromagnetic and valance bond fluctuations are critical. In the regime (A) contributions from superspin fluctuations exhibit an exponential dependence of temperature and ignored in the low energy limit. Dominant contributions are driven by $z=3$ critical gauge fluctuations, resulting in $\gamma(T) \sim T^{-1 / 3}$. In the regime (B) antiferromagnetic fluctuations cause an exponential dependence of temperature while both valance bond and gauge fluctuations give rise to $\gamma(T) \sim T^{-1 / 3}$ due to their $z=3$ criticality. In the regime (C) $z=3$ critical valance bond excitations and gauge fluctuations allow $\gamma(T) \sim T^{-1 / 3}$ while $z=1$ critical antiferromagnetic fluctuations result in $\gamma_{A F}\left(T>\frac{\xi^{-1}}{2}\right)=\frac{6}{\pi}\left(\int_{0}^{\infty} d x \frac{x^{3}}{\sinh ^{2} x}\right) T$, sub-leading thus ignored in the low energy limit.

\section{F. Transport}

We discuss electrical transport of the effective field theory Eq. (10). As shown in the Eliashberg equations (17), the holon self-energy is given by two contributions

$$
\begin{aligned}
& \Sigma_{\eta}^{v}(i \omega)=m_{\eta}^{2} T \sum_{i \Omega} \int \frac{d^{d} q}{(2 \pi)^{d}} \\
& \sum_{m, n=4}^{5} D_{v}^{m n}(q, i \Omega) \gamma_{m} G_{\eta}(k+q, i \omega+i \Omega) \gamma_{n}, \\
& \Sigma_{\eta}^{a}(i \omega)=T \sum_{i \Omega} \int \frac{d^{d} q}{(2 \pi)^{d}} \\
& D_{a}^{\mu \nu}(q, i \Omega) \gamma_{\mu} \tau_{3} G_{\eta}(k+q, i \omega+i \Omega) \gamma_{\nu} \tau_{3},
\end{aligned}
$$

where the first is due to valance bond or monopole fluctuations, and the second comes from gauge fluctuations.

We first consider the self-energy due to valance bond fluctuations. The above expression can be written as follows

$$
\begin{aligned}
& \Sigma_{\eta}^{v}(i \omega) \approx-\frac{2 g m_{\eta}^{2}}{\mu_{h}} T \sum_{i \Omega} \int \frac{d^{d} q}{(2 \pi)^{d}} \\
& \frac{1}{q^{2}+\xi^{-2}+\gamma_{v} \frac{|\Omega|}{q}} \frac{i \gamma_{0} \omega+i \gamma_{i} k_{i}^{F}+\mu_{h} \gamma_{0}}{i \omega+i \Omega-q \cos \theta},
\end{aligned}
$$

where the self-energy correction of doped holes via $z=3$ valance bond fluctuations is clearly seen. Here, $k_{F}=$ $\sqrt{k_{x}^{F 2}+k_{y}^{F 2}}=\mu_{h}$ is the holon Fermi momentum. Then, the imaginary part of the self-energy is given by

$$
\begin{aligned}
& \Im \Sigma_{\eta}^{v}(\omega+i \delta)=\frac{g m_{\eta}^{2}}{2 \pi^{3} \mu_{h}}\left[\gamma_{0} \omega+i \gamma_{i} k_{i}^{F}+\mu_{h} \gamma_{0}\right] \\
& \int_{0}^{|\omega|} d \Omega_{1} \int_{\xi^{-1}}^{\infty} d q \frac{q}{\sqrt{q^{2}-\left(\omega+\Omega_{1}\right)^{2}}} \frac{\gamma_{v} \Omega_{1} q}{q^{6}+\gamma_{v}^{2} \Omega_{1}^{2}}
\end{aligned}
$$

where Wick rotation is performed at zero temperature in order to see frequency dependence of the self-energy.

Performing momentum and frequency integrals, we find

$$
\begin{aligned}
& \Im \Sigma_{\eta}^{v}\left(\omega>\frac{\left(\gamma_{v} \xi^{3}\right)^{-1}}{2}\right) \approx \frac{g m_{\eta}^{2}}{4 \sqrt{3} \pi^{2} \gamma_{v}^{1 / 3}} \gamma_{0}|\omega|^{2 / 3} \\
& \Im \Sigma_{\eta}^{v}\left(\omega<\frac{\left(\gamma_{v} \xi^{3}\right)^{-1}}{2}\right) \approx \frac{g m_{\eta}^{2} \xi}{\sqrt{3} \pi^{2}} \gamma_{0} \omega+\gamma_{0} \mathcal{O}\left(\omega^{2}\right) .
\end{aligned}
$$

Note that the $|\omega|^{2 / 3}$ behavior is the hallmark of $z=3$ criticality in two dimensions. 26] The self-energy correction due to gauge fluctuations also gives rise to $\Im \Sigma_{\eta}^{a}(\omega) \propto$ $|\omega|^{2 / 3}$.

At finite temperatures the zero-frequency self-energy corrections turn out to diverge in the one-loop approximation. However, such divergences due to both gauge and valance bond fluctuations need not be given 
much attention because such self-energies are not gaugeinvariant, thus they do not have any physical meaning. These divergences should be considered as an artifact of gauge non-invariance. Gauge invariance can be incorporated via vertex corrections, which cancel the divergent parts in the self-energies, giving rise to gauge invariant finite contributions. [27, 28, 29] This corresponds to the transport time, given by $q^{2} \sim T^{\frac{2}{z}}$ multiplication in the quasiparticle life time. As a result, we find the following expression for the electrical resistivity

$$
\rho(T) \propto T^{4 / 3}
$$

consistent with the previous results. [26] We obtain nonFermi liquid physics in both quantum critical and disordered phases, where both valance bond and gauge fluctuations cause the non-Fermi liquid transport in the quantum critical regime while only gauge fluctuations result in that in the disordered phase.

\section{APPLICATION TO ONE DIMENSION}

Although our effective field theory Eq. (10) is physically reasonable, we would like to justify its validity applying it to one dimension. In one dimension the spin sector is described by the $\mathrm{SO}(4)$ WZW theory, and the charge sector is represented by $\mathrm{QED}_{2}$ without the chemical potential term. Accordingly, the coupling term between valance bond fluctuations and holons is adjusted. The resulting effective field theory in one dimension is obtained to be

$$
\begin{aligned}
& S=\int d^{2} x\left[\frac{1}{2 g} \sum_{k=1}^{4}\left(\partial_{\mu} v_{k}\right)^{2}\right. \\
& \left.+i \frac{2 \pi}{\operatorname{Area}\left(S^{3}\right)} \int_{0}^{1} d t \epsilon_{a b c d} v_{a} \partial_{t} v_{b} \partial_{\tau} v_{c} \partial_{x} v_{d}\right] \\
& +\int d^{2} x\left[\bar{\eta} \gamma_{\mu}\left(\partial_{\mu}-i a_{\mu}^{3} \tau_{3}-i A_{\mu}\right) \eta-m_{\eta} \bar{\eta}\left(i \gamma_{5} v_{4}\right) \eta\right. \\
& \left.+\frac{1}{2 e^{2}}\left(\epsilon_{3 \mu \nu} \partial_{\mu} a_{\nu}^{3}\right)^{2}\right],
\end{aligned}
$$

where $\gamma_{\mu}$ and $\gamma_{5}$ are $2 \times 2$ matrices, and $\eta$ is a four component Dirac spinor. The SO(4) WZW theory of the spin sector has been derived in Ref. [12], using the path integral formulation for non-abelian chiral anomaly. Here, we investigate the role of massless Dirac fermions, introduced from Eq. (10) by reducing the two dimensional theory down to one dimension.

Performing the abelian bosonization for the fermion sector, we obtain the following expression

$$
\begin{aligned}
& S=\int d^{2} x\left[\frac{1}{2 g} \sum_{k=1}^{4}\left(\partial_{\mu} v_{k}\right)^{2}\right. \\
& \left.+i \frac{2 \pi}{\operatorname{Area}\left(S^{3}\right)} \int_{0}^{1} d t \epsilon_{a b c d} v_{a} \partial_{t} v_{b} \partial_{\tau} v_{c} \partial_{x} v_{d}\right] \\
& +\int d^{2} x\left[\frac{1}{2}\left(\partial_{\mu} \phi_{+}\right)^{2}+\frac{1}{2}\left(\partial_{\mu} \phi_{-}\right)^{2}\right. \\
& +\left(\frac{\Lambda}{\pi} m_{\eta}\right) v_{4} \sin \left(\sqrt{4 \pi} \phi_{+}\right)+\left(\frac{\Lambda}{\pi} m_{\eta}\right) v_{4} \sin \left(\sqrt{4 \pi} \phi_{-}\right) \\
& -i a_{\mu}^{3}\left(\frac{1}{2 \pi} \epsilon_{\mu \nu} \partial_{\nu} \phi_{+}-\frac{1}{2 \pi} \epsilon_{\mu \nu} \partial_{\nu} \phi_{-}\right) \\
& \left.-i A_{\mu}\left(\frac{1}{2 \pi} \epsilon_{\mu \nu} \partial_{\nu} \phi_{+}+\frac{1}{2 \pi} \epsilon_{\mu \nu} \partial_{\nu} \phi_{-}\right)+\frac{1}{2 e^{2}}\left(\epsilon_{\mu \nu} \partial_{\mu} a_{\nu}^{3}\right)^{2}\right]
\end{aligned}
$$

where the subscript \pm in the bosonic field $\phi_{ \pm}$represents the $\mathrm{SU}(2)$ doublet of $\tau_{3}$, and $\Lambda$ is a cutoff associated with band linearization.

Performing integration for $\mathrm{U}(1)$ gauge fields, we find a mass-type term $\frac{e^{2}}{8 \pi^{2}}\left(\phi_{+}-\phi_{-}\right)^{2}$. This allows us to set $\phi_{+}=\phi_{-} \equiv \phi$ in the low energy limit. Shifting $\sqrt{4 \pi} \phi$ with $-\frac{\pi}{2}+\sqrt{4 \pi} \theta$, we are led to

$$
\begin{aligned}
& S=\int d^{2} x\left[\frac{1}{2 g} \sum_{k=1}^{4}\left(\partial_{\mu} v_{k}\right)^{2}\right. \\
& \left.+i \frac{2 \pi}{\operatorname{Area}\left(S^{3}\right)} \int_{0}^{1} d t \epsilon_{a b c d} v_{a} \partial_{t} v_{b} \partial_{\tau} v_{c} \partial_{x} v_{d}\right] \\
& +\int d^{2} x\left[\left(\partial_{\mu} \theta\right)^{2}-\left(\frac{2 \Lambda}{\pi} m_{\eta}\right) v_{4} \cos (\sqrt{4 \pi} \theta)\right. \\
& \left.-i A_{\mu}\left(\frac{1}{\pi} \epsilon_{\mu \nu} \partial_{\nu} \theta\right)\right] .
\end{aligned}
$$

It is interesting to see that valance bond excitations lead to charge density wave fluctuations, consistent with our expectation.

The valance bond and charge density wave coupling term can be taken into account in the cumulant expansion, and the correction part is given by

$$
\begin{aligned}
& \delta S=-\frac{1}{2}\left(\left\langle S_{\text {int }}^{2}\right\rangle-\left\langle S_{\text {int }}\right\rangle^{2}\right) \\
& =-\frac{1}{2}\left(\frac{2 \Lambda}{\pi} m_{\eta}\right)^{2} \int d^{2} x \int d^{2} x^{\prime} \\
& \left\{v_{4}(x)\left\langle\cos (\sqrt{4 \pi} \theta(x)) \cos \left(\sqrt{4 \pi} \theta\left(x^{\prime}\right)\right)\right\rangle v_{4}\left(x^{\prime}\right)\right. \\
& \left.+\cos (\sqrt{4 \pi} \theta(x))\left\langle v_{4}(x) v_{4}\left(x^{\prime}\right)\right\rangle \cos \left(\sqrt{4 \pi} \theta\left(x^{\prime}\right)\right)\right\} \\
& \equiv \delta S_{v_{4}}+\delta S_{\theta},
\end{aligned}
$$

where $S_{i n t}=-\int d^{2} x\left(\frac{2 \Lambda}{\pi} m_{\eta}\right) v_{4} \cos (\sqrt{4 \pi} \theta)$ is the coupling term.

It is not difficult to evaluate the density-density correlation function since charge fluctuations are described by 
the noninteracting Gaussian ensemble if metallic charge dynamics is assumed. In this case we find

$$
\begin{aligned}
& \left\langle\cos (\sqrt{4 \pi} \theta(x)) \cos \left(\sqrt{4 \pi} \theta\left(x^{\prime}\right)\right)\right\rangle \propto \cosh \left(4 \pi\left\langle\theta(x) \theta\left(x^{\prime}\right)\right\rangle\right) \\
& =\cosh \left(4 \pi \mathcal{C}_{\theta} \ln \left|x-x^{\prime}\right|\right) \rightarrow\left|x-x^{\prime}\right|^{4 \pi \mathcal{C}_{\theta}},
\end{aligned}
$$

where $\mathcal{C}_{\theta}$ is a positive numerical constant, and the last part is valid at large distances, i.e., $\left|x-x^{\prime}\right| \rightarrow \infty$.

Inserting this expression into the spin sector, we obtain an effective theory for $\mathrm{SO}(4)$ spin fluctuations

$$
\begin{aligned}
& S_{v_{4}}=\int d^{2} x\left[\frac{1}{2 g} \sum_{k=1}^{4}\left(\partial_{\mu} v_{k}\right)^{2}\right. \\
& \left.+i \frac{2 \pi}{\operatorname{Area}\left(S^{3}\right)} \int_{0}^{1} d t \epsilon_{a b c d} v_{a} \partial_{t} v_{b} \partial_{\tau} v_{c} \partial_{x} v_{d}\right] \\
& -\int d^{2} x \int d^{2} x^{\prime} \mathcal{C}_{v_{4}}\left(\frac{2 \Lambda}{\pi} m_{\eta}\right)^{2} v_{4}(x)\left|x-x^{\prime}\right|^{4 \pi \mathcal{C}_{\theta}} v_{4}\left(x^{\prime}\right),
\end{aligned}
$$

where $\mathcal{C}_{v_{4}}$ is a positive numerical constant. An important point is that metallic charge fluctuations give rise to confining interactions between monopoles, suppressing monopole fluctuations. This tendency is completely consistent with our previous two dimensional analysis, where holon fluctuations cause dissipative monopole dynamics described by $z=3$, prohibiting their proliferation. In one dimension charge dynamics suppresses monopole condensation more strongly.

An immediate question is the nature of spin dynamics descried by Eq. (41). Since monopole excitations will be suppressed via charge fluctuations, spinon deconfinement is expected to appear. A relevant point is whether spin fluctuations are critical or not. The $\mathrm{SO}(4)$ WZW theory is well known to exhibit criticality without the fermion-induced monopole-suppressing term at half filling, where monopole excitations turn out to be irrelevant due to the presence of the topological WZW term, which differs completely from the charge-dynamics monopolesuppressing mechanism. Since the $\mathrm{SO}(4)$ symmetry is broken by the presence of charge fluctuations shown by the last term of Eq. (41), we expect that the WZW term may not be relevant in this case, thus resulting in spin gap. This seems to be consistent with our physical intuition that charge fluctuations will cut spin correlations, making their correlation length short. Then, the resulting state is identified with the Luther-Emery phase, where spin fluctuations are gapped and charge excitations exhibit enhanced superconducting correlations. 30.

On the other hand, if charge fluctuations are gapped, i.e., in the Mott insulating phase, their density-density correlations will vanish at large distances as follows, $\left\langle\cos (\sqrt{4 \pi} \theta(x)) \cos \left(\sqrt{4 \pi} \theta\left(x^{\prime}\right)\right)\right\rangle \propto e^{-\left|x-x^{\prime}\right| / \xi_{\eta}}$, where $\xi_{\eta}^{-1}$ is associated with their excitation gap. Then, spin dynamics will be described by the pure SO(4) WZW theory in the long wave-length limit. As a result, the critical spin liquid Mott insulator is expected to appear in this case.

\section{SUPERCONDUCTIVITY AND STABILITY OF THE ANOMALOUS METAL}

Until now, we have seen the nature of the anomalous metallic phase. An important problem is how d-wave superconductivity evolves from the non-Fermi liquid state. In our effective theory approach superconductivity can appear only from pairing of fermionic doped holes. As shown explicitly in our effective action Eq. (10), interactions between doped holes are mediated by both gauge and valance bond fluctuations. An important thing is that these interactions compete with each other.

Since holons carry an isospin quantum number represented by the $\tau_{3}$ matrix, two kinds of fermion pairs can be considered. When their isospin quantum numbers are different from each other, gauge fluctuations cause attractive interactions for the particle-particle channel while valance bond fluctuations give rise to repulsive ones. This is nothing but the pairing possibility between $b_{1}$ and $b_{2}$ bosons in the bosonic description [14] of the SU(2) slaveboson theory, discussed by Wen and Lee. [3] In this respect such pairing possibility is the unique feature of the $\mathrm{SU}(2)$ slave-boson description for superconductivity.

On the other hand, when their isospin quantum numbers are the same as each other, gauge fluctuations give rise to repulsive interactions for the particle-particle channel while valance bond fluctuations cause attractive ones between holons on different sublattices. Do not confuse the present uniform (ferromagnetic) gauge fluctuations with staggered (antiferromagnetic) ones. Staggered gauge fluctuations, discussed previously in the slavefermion context, cause attractive interactions between doped holes on different sublattices. Gauge fluctuations appearing in the present theory are in the uniform channel associated with the third component of the $\mathrm{SU}(2)$ slave-boson theory. The presence of $\tau_{3}$ matrix in the gauge coupling supports this fact. As a result, such holons have the same gauge charges, repulsing each other.

Based on the above discussion, we conclude that emergence of superconductivity is determined by the relative strength between the couplings of gauge and valance bond fluctuations with doped holes. An interesting observation is that the presence of repulsive interactions in both cases may favor d-wave pairing of doped holes.

Now, we derive a full set of Eliashberg equations including the pairing channel. In this paper we consider only the first case, that is, pairing between different isospins. One can construct the Luttinger-Ward func- 
tional in the same way as the previous case Eq. (16),

$$
\begin{aligned}
& F_{L W}=F_{L W}^{\eta}+F_{L W}^{v}+F_{L W}^{a}+Y_{v}+Y_{a}, \\
& F_{L W}^{\eta}=-T \sum_{i \omega} \int \frac{d^{d} k}{(2 \pi)^{d}} \operatorname{tr}\left[\ln \left\{g_{\eta}^{-1}(k, i \omega) \mathbf{I}+\boldsymbol{\Sigma}_{\eta}(i \omega)\right\}\right. \\
& \left.-\boldsymbol{\Sigma}_{\eta}(i \omega) \mathbf{G}_{\eta}(k, i \omega)\right] \\
& F_{L W}^{v}=T \sum_{i \Omega} \int \frac{d^{d} q}{(2 \pi)^{d}}\left[\sum _ { m , n = 1 } ^ { 5 } \operatorname { l n } \left\{d_{v}^{-1}(q, i \Omega) \delta_{m n}\right.\right. \\
& \left.\left.+\Pi_{v}^{m n}(q, i \Omega)\right\}-\sum_{m, n=1}^{5} \Pi_{v}^{m n}(q, i \Omega) D_{v}^{m n}(q, i \Omega)\right]-m_{v}^{2}, \\
& F_{L W}^{a}=T \sum_{i \Omega} \int \frac{d^{d} q}{(2 \pi)^{d}}\left[\ln \left\{d_{a}^{-1}(q, i \Omega)+\Pi_{a}(q, i \Omega)\right\}\right. \\
& \left.-\Pi_{a}(q, i \Omega) D_{a}(q, i \Omega)\right], \\
& Y_{v}=-\frac{m_{\eta}^{2}}{2} T \sum_{i \omega} \int \frac{d^{d} k}{(2 \pi)^{d}} T \sum_{i \Omega} \int \frac{d^{d} q}{(2 \pi)^{d}} \\
& \sum_{m, n=4}^{5} \operatorname{tr}\left[D_{v}^{m n}(q, i \Omega) \gamma_{m} \mathbf{G}_{\eta}(k+q, i \omega+i \Omega) \gamma_{n} \mathbf{G}_{\eta}(k, i \omega)\right], \\
& Y_{a}=-\frac{1}{2} T \sum_{i \omega} \int \frac{d^{d} k}{(2 \pi)^{d}} T \sum_{i \Omega} \int \frac{d^{d} q}{(2 \pi)^{d}} \\
& \operatorname{tr}\left[D_{a}^{\mu \nu}(q, i \Omega) \gamma_{\mu} \tau_{3} \mathbf{G}_{\eta}(k+q, i \omega+i \Omega) \gamma_{\nu} \tau_{3} \mathbf{G}_{\eta}(k, i \omega)\right](42)
\end{aligned}
$$

Here, $\mathbf{G}_{\eta}{ }^{-1}(k, i \omega)=\mathbf{g}^{-1}(k, i \omega)+\boldsymbol{\Sigma}(k, i \omega)$ is the matrix green's function with an anomalous propagator, where $\mathbf{g}(k, i \omega)=g(k, i \omega) \mathbf{I}$ with $g^{-1}(k, i \omega)=\left(i \gamma_{\mu} k_{\mu}+\right.$ $\left.\mu_{h} \gamma_{0}\right)^{-1}$ is the bare green's function and $\boldsymbol{\Sigma}(k, i \omega)=$ $\left(\begin{array}{cc}\Sigma(k, i \omega) & \Delta(k, i \omega) \\ \Delta^{*}(k, i \omega) & \Sigma(k, i \omega)\end{array}\right)$ is the self-energy matrix with its anomalous part.

The above Luttinger-Ward functional results in the following Eliashberg equations

$$
\begin{aligned}
& \boldsymbol{\Sigma}_{\eta}(i \omega)=m_{\eta}^{2} T \sum_{i \Omega} \int \frac{d^{d} q}{(2 \pi)^{d}} \sum_{m, n=4}^{5} \\
& D_{v}^{m n}(q, i \Omega) \gamma_{m} \mathbf{G}_{\eta}\left(k_{F}+q, i \omega+i \Omega\right) \gamma_{n}+T \sum_{i \Omega} \int \frac{d^{d} q}{(2 \pi)^{d}} \\
& D_{a}^{\mu \nu}(q, i \Omega) \gamma_{\mu} \tau_{3} \mathbf{G}_{\eta}\left(k_{F}+q, i \omega+i \Omega\right) \gamma_{\nu} \tau_{3}, \\
& \Pi_{v}^{m n}(q, i \Omega)=T \sum_{i \omega} \int \frac{d^{d} k}{(2 \pi)^{d}} \\
& \sum_{m, n=4}^{5}\left(-\frac{m_{\eta}^{2}}{2} \operatorname{tr}\left[\gamma_{m} \mathbf{G}_{\eta}(k+q, i \omega+i \Omega) \gamma_{n} \mathbf{G}_{\eta}(k, i \omega)\right]\right), \\
& \Pi_{a}^{\mu \nu}(q, i \Omega)=T \sum_{i \omega} \int \frac{d^{d} k}{(2 \pi)^{d}} \\
& \left(-\frac{1}{2} \operatorname{tr}\left[\gamma_{\mu} \tau_{3} \mathbf{G}_{\eta}(k+q, i \omega+i \Omega) \gamma_{\nu} \tau_{3} \mathbf{G}_{\eta}(k, i \omega)\right]\right),
\end{aligned}
$$

where this is basically the same as the previous one Eq.
(17), but the holon propagator is replaced with a matrix including its superconducting part. If we rewrite the above equations with each component of the holon propagator, we obtain

$$
\begin{aligned}
& \Sigma(k, i \omega) \\
& =m_{\eta}^{2} T \sum_{i \Omega, q} \sum_{n=3}^{4} \gamma_{n} G(k+q, i \omega+i \Omega) D_{n n}^{v}(q, i \Omega) \gamma_{n} \\
& +T \sum_{i \Omega, q} \sum_{i, j=x, y} \gamma_{i} G(k+q, i \omega+i \Omega) D_{i j}^{a}(q, i \Omega) \gamma_{j}
\end{aligned}
$$

and

$$
\begin{aligned}
& \Delta(k, i \omega) \\
& =m_{\eta}^{2} T \sum_{i \Omega, q} \sum_{n=3}^{4} \gamma_{n} F(k+q, i \omega+i \Omega) D_{n n}^{v}(q, i \Omega) \gamma_{n} \\
& -T \sum_{i \Omega, q} \sum_{i, j=x, y} \gamma_{i} F(k+q, i \omega+i \Omega) D_{i j}^{a}(q, i \Omega) \gamma_{j},
\end{aligned}
$$

where the normal and abnormal holon propagators are given by

$$
\begin{aligned}
& G(k, i \omega)=\frac{g^{-1}(k, i \omega)+\Sigma(k, i \omega)}{\left[g^{-1}(k, i \omega)+\Sigma(k, i \omega)\right]^{2}-|\Delta(k, i \omega)|^{2}}, \\
& F(k, i \omega)=-\frac{\Delta(k, i \omega)}{\left[g^{-1}(k, i \omega)+\Sigma(k, i \omega)\right]^{2}-|\Delta(k, i \omega)|^{2}} .
\end{aligned}
$$

As shown in Eqs. (45) and (46), gauge fluctuations cause attractive interactions between holons with different isospins, guaranteed by the the presence of the $\tau_{3}$ matrix, while valance bond fluctuations result in repulsive ones. The presence of repulsive interactions opens the possibility of d-wave pairing of doped holes.

As discussed above, there is another pairing channel between holons with same isospins but on different sublattices, induced by valance bond fluctuations. Comparison of these two superconducting phases by solving each Eliashberg equations remains as an important future work. What we would like to mention is that because both gauge and valance bond fluctuations give rise to competing interactions, the present anomalous metallic phase is expected to be stable against superconductivity in some parameter regions of the effective theory Eq. (10). Even if such a metallic phase becomes unstable against superconductivity at zero temperature, at finite temperatures the present non-Fermi liquid physics would survive.

There is another instability channel associated with particle-hole pairing. Different from the particle-particle channel, gauge fluctuations cause attractive interactions for the particle-hole channel when holons have the same isospin. As pointed out by Altshuler et al.,[31] the $2 k_{F}$ vertex with the Fermi momentum $k_{F}$ is power-law diverging with its exponent $1 / N$ approximately, where $N$ is the flavor number of fermions. They showed that divergence of particle-hole susceptibility depends on the exponent 
of the diverging vertex. In other words, the particle-hole pairing instability arises when the divergence of the $2 k_{F}$ interaction vertex is sufficiently strong. In this respect the present effective theory with the large $N$ approximation is expected to be free from such particle-hole instabilities. Remember that the large $N$ approximation is consistent with the self-consistent Eliashberg framework. Interestingly, valance bond fluctuations are against such particle-hole pairings as they compete with gauge fluctuations for superconductivity. More quantitative analysis for the particle-hole channel is required when the flavor number of fermions is not large. However, this is beyond the scope of the present paper since it is not clear whether even the Eliashberg approximation is stable or not in this case.

In the recent publication 32 the presence of an interesting inhomogeneous superconducting state was demonstrated in the two-leg ladder system based on the renormalized mean-field theory, where a heavy numerical analysis was performed for solving self-consistent mean-field equations of order parameters in real space. From the present approach it is difficult to see the emergence of such complicated inhomogeneous order parameter patterns because our effective field theory is based on the uniform phase of order parameters.

Applying the present theoretical framework to the twoleg ladder system, one would see that gauge fluctuations enhance the $2 k_{F}$ particle-hole vertex to cause power-law divergence, but the particle-hole susceptibility does not diverge at least in the large $N$ approximation, as discussed above. However, charge and spin density waves can certainly occur when the fermion flavor number is small. In this respect, when the coupling strength between doped holes and valance bond fluctuations is large but the fermion flavor number is not large, an inhomogeneous superconducting phase is expected to appear. Unfortunately, fully self-consistent analysis including both particle-particle and particle-hole instabilities is clearly beyond the scope of the present paper.

\section{SUMMARY}

Fermionizing the charge sector and bosonizing the spin part in the $\mathrm{SU}(2)$ slave-boson theory, we have derived an effective field theory for dynamics of doped holes in the antiferromagnetically correlated spin background, where spin fluctuations are described by the $\mathrm{SO}(5)$ WZW theory while charge dynamics is expressed by non-relativistic $\mathrm{QED}_{3}$ around four Dirac nodes. In particular, hole dynamics affects deconfinement of bosonic spinons in the $\mathrm{SO}(5)$ WZW theory through the coupling term between valance bond (monopole) fluctuations and fermionic holons. Such interactions give rise to $z=3$ criticality for monopole dynamics, prohibiting their proliferation in the presence of the WZW term. As a result, holon fluctuations turn out to help spin fractionalization near the quantum critical point.
We have investigated thermodynamics and transport in the Eliashberg framework for our effective field theory. We pointed out that the Eliashberg framework is the lowest order self-consistent approximation well controlled in our effective field theory, where Migdal theorem works well owing to $z=3$ criticality, and the large $N_{\eta}$ limit with the number of Dirac nodes $N_{\eta}$ is naturally allowed. We find that spin fluctuations are described by $z=1$ for antiferromagnetic fluctuations and $z=3$ for valance bond excitations, giving rise to three regimes, where superspin fluctuations are gapped at low temperatures, only valance bond excitations are critical at intermediate temperatures, and superspin fluctuations are critical at high temperatures. Both valance bond and gauge fluctuations are described by $z=3$ critical theory, and we find non-Fermi liquid physics for thermodynamics and electrical transport near the quantum critical point, consistent with $z=3$ scaling. In addition, even in the quantum disordered phase such non-Fermi liquid physics is preserved owing to critical gauge fluctuations.

To further justify our effective field theory, we have applied it to one dimension, physically well known. In one dimension spin fluctuations are described by the $\mathrm{SO}(4)$ WZW theory while charge excitations are represented by $\mathrm{QED}_{2}$. We have taken the monopole-holon coupling term into account in the abelian bosonization framework. We demonstrated that holon dynamics results in confining interactions between monopole excitations. Thus, we conclude that charge fluctuations help spinon deconfinement in both one and two dimensional cases while charge dynamics suppresses monopole fluctuations more strongly in one dimension.

We have discussed stability of the non-Fermi liquid metallic phase against superconductivity and density waves. An interesting observation is that two kinds of holon pairing channels exist due to the presence of the isospin quantum number in the $\mathrm{SU}(2)$ slave-boson description. In the different isospin channel attractive pairing interactions are caused by gauge fluctuations while in the same isospin channel such interactions arise from valance bond fluctuations. An important thing is that such gauge and valance bond interactions compete with each other. As a result, superconductivity is expected to appear in a limited parameter range. The presence of repulsive interactions allows the possibility of d-wave pairing of doped holes. For the particle-hole channel, we argued that as far as the fermion flavor number is sufficiently large, consistent with the Eliashberg approximation, the homogeneous metallic phase can be stable against charge and spin density waves.

We would like to emphasize that the SU(2) structure is important for our treatment. If we start from the $\mathrm{U}(1)$ slave-boson representation, the fermionization procedure is not performed naturally since we have nonzero net flux owing to the presence of finite density of holons. Furthermore, the SO(5) WZW theory for the spin sector does not appear owing to the contribution from a chemical potential term in the $\mathrm{U}(1)$ slave-boson framework. 
Our effective field theory exhibits direct interactions between monopoles and holes, associated with deconfinement of bosonic spinons away from half filling. The important issue how doped holes affect spinon deconfinement deserves to be studied more carefully.

We appreciate extremely helpful discussions with
A. Tanaka for his SO(5) WZW description. This work is supported by the French National Grant ANR36ECCEZZZ. K.-S. Kim is also supported by the Korea Research Foundation Grant (KRF-2007-357C00021) funded by the Korean Government.
[1] A. Damascelli, Z.-X. Shen, Z. Hussain, Rev. Mod. Phys. 75, 473 (2003), and references therein.

[2] T. Timusk and B.W. Statt, Rep. Prog. Phys. 62, 61 (1999), and references therein.

[3] P. A. Lee, N. Nagaosa, and X.-G. Wen, Rev. Mod. Phys. 78, 17 (2006), and references therein.

[4] The U(1) slave-boson formulation cannot explain the doping independent decreasing ratio of superfluid weight owing to charge renormalization of nodal quasiparticles as a function of hole concentration. [3] In addition, it cannot explain the existence of coherent electron excitations near the nodal points in the anomalous normal state although instanton physics of U(1) gauge fields is argued to cause confinement and result in such excitations. 3]

[5] B. A. Bernevig, D. Giuliano, and R. B. Laughlin, Annals Phys. 311, 182 (2004).

[6] T. Senthil, A. Vishwanath, L. Balents, S. Sachdev, and M. P. A. Fisher, Science 303, 1490 (2004); T. Senthil, L. Balents, S. Sachdev, A. Vishwanath, and M. P.A. Fisher, Phys. Rev. B 70, 144407 (2004).

[7] Ki-Seok Kim, Phys. Rev. B 72, 035109 (2005); This issue was investigated in detail.

[8] A. W. Sandvik, Phys. Rev. Lett. 98, 227202 (2007).

[9] M. Hermele, T. Senthil, and M. P. A. Fisher, Phys. Rev. B 72, 104404 (2005).

[10] Y. Ran and X.-G. Wen, arXiv:cond-mat/0609620v3 (unpublished).

[11] A. Tanaka and X. Hu, Phys. Rev. Lett. 95, 036402 (2005).

[12] A. Tanaka and X. Hu, Phys. Rev. Lett. 88, 127004 (2002).

[13] S. Sachdev, Nature Physics 4, 173 (2008), and references therein.

[14] P. A. Lee, N. Nagaosa, T.-K. Ng, and X.-G. Wen, Phys. Rev. B 57, 6003 (1998); A bosonic field theoretic description for the holon sector has been pursued. Inserting the following expression $h_{i}^{S F}=(\sqrt{x}+$ $\left.\delta b_{i}\right)\left(\begin{array}{c}z_{i 1} \\ -i(-1)^{i_{x}+i_{y}} z_{i 2}\end{array}\right)$ with $z_{i 1}=e^{i \alpha_{i}} e^{-i \frac{\phi_{i}}{2}} \cos \frac{\theta_{i}}{2}$ and $z_{i 2}=e^{i \alpha_{i}} e^{i \frac{\phi_{i}}{2}} \sin \frac{\theta_{i}}{2}$ into the holon sector of the $\mathrm{SU}(2)$ slave-boson theory, where this representation can be derived easily from an $\mathrm{SU}(2)$ rotation of $h_{i}^{S F}=g_{i}^{\dagger} h_{i}^{d S C}$ with an $\mathrm{SU}(2)$ matrix field $g_{i}^{\dagger}=\exp \left[-i(-1)^{i_{x}+i_{y}} \frac{\theta_{i}}{2} \tau_{1}\right] \exp \left[-i \frac{\phi_{i}}{2} \tau_{3}\right]$ and $h_{i}^{d S C}=$ $\left(\begin{array}{c}\sqrt{x} \\ 0\end{array}\right)$, and performing integration of amplitude fluc- tuations $\delta b_{i}$, we find an effective field theory

$$
\begin{aligned}
& \mathcal{L}_{h}=x \mathbf{z}^{\dagger}\left(\partial_{\tau}-i a_{0}^{3} \tau_{3}-i A_{0}\right) \mathbf{z}+\frac{1}{u_{h}}\left|\mathbf{z}^{\dagger}\left(\partial_{\tau}-i a_{0}^{3} \tau_{3}-i A_{0}\right) \mathbf{z}\right|^{2} \\
& +\frac{x}{2 m_{b}}\left|\left(\partial_{i}-i a_{i}^{3} \tau_{3}-i A_{i}\right) \mathbf{z}\right|^{2} \\
& +x^{2} J\left[\frac{4}{c_{1}}\left|z_{1}\right|^{2}\left|z_{2}\right|^{2}+\frac{1}{c_{2}}\left(\left|z_{1}\right|^{2}-\left|z_{2}\right|^{2}\right)^{2}\right]
\end{aligned}
$$

where $u_{h}$ is associated with compressibility for holons, and $m_{b} \sim 1 / t$ is bare band mass. $c_{1}$ and $c_{2}$ are positive numerical constants associated with anisotropy for the holon isospin $\vec{I}_{h i}=z_{i \alpha}^{\dagger} \vec{\tau}_{\alpha \beta} z_{i \beta}$.

[15] J. Rech, C. Pepin, and A. V. Chubukov, Phys. Rev. B 74, 195126 (2006).

[16] R. K. Kaul, Y. B. Kim, S. Sachdev, and T. Senthil, Nature Physics 4, 28 (2008).

[17] K.-S. Kim and M. D. Kim, Phys. Rev. B 77, 125103 (2008); K.-S. Kim and Mun Dae Kim, Phys. Rev. B 75, 035117 (2007).

[18] N. Read and S. Sachdev, Phys. Rev. B 42, 4568 (1990); S. Sachdev and N. Read, Int. J. Mod. Phys. B 5, 219 (1991).

[19] C. Xu and S. Sachdev, Phys. Rev. Lett. 100, 137201 (2008).

[20] A. G. Abanov and P. B. Wiegmann, Nucl. Phys. B 570, 685 (2000).

[21] Ki-Seok Kim, Phys. Rev. B 72, 214401 (2005).

[22] S. Ryu, O. I. Motrunich, J. Alicea, and Matthew P. Fisher, Phys. Rev. B 75, 184406 (2007).

[23] A. V. Chubukov, Phys. Rev. B 72, 085113 (2005).

[24] A. V. Chubukov, D. L. Maslov, S. Gangadharaiah, and L. I. Glazman, Phys. Rev. B 71, 205112 (2005); See appendix D.

[25] In the pure $\mathrm{SO}(5)$ nonlinear $\sigma$ model we find

$$
1=\frac{5 g}{\pi} T\left\{\ln \sinh \left(\frac{\Lambda}{2 T}\right)-\ln \sinh \left(\frac{\xi_{v}^{-1}}{2 T}\right)\right\}
$$

for the correlation length. Its solution is given by

$$
\begin{aligned}
& \xi_{v}^{-1}\left(g \sim g_{c}^{v} ; T<\frac{\xi_{v}^{-1}}{2}\right) \approx \Lambda-\frac{2 \pi}{5 g} \equiv \frac{2 \pi}{5}\left(\frac{1}{g_{c}^{v}}-\frac{1}{g}\right), \\
& \xi_{v}^{-1}\left(g \sim g_{c}^{v} ; T>\frac{\xi_{v}^{-1}}{2}\right)=2 T e^{\frac{\pi}{5} \frac{\left(\frac{1}{g_{c}^{v}}-\frac{1}{g}\right)}{T}},
\end{aligned}
$$

resulting in $g_{c}^{v}>g_{c}$, consistent with our intuition.

[26] P. A. Lee and N. Nagaosa, Phys. Rev. B 46, 5621 (1992); L. B. Ioffe and G. Kotliar, Phys. Rev. B 42, 10348 (1990).

[27] Y. Nambu, Phys. Rev. 117, 648 (1960).

[28] Y.-B. Kim, A. Furusaki, X.-G. Wen, and P. A. Lee, Phys. Rev. B 50, 17917 (1994)

[29] D. H. Kim and P. A. Lee, Annals Phys. 272, 130 (1999). 
[30] N. Nagaosa, Quantum Field Theory in Strongly Correlated Electronic Systems (Springer-Verlag, Berlin, 1999).

[31] B. L. Altshuler, L. B. Ioffe, and A. J. Millis, Phys. Rev. B 50, 14048 (1994).
[32] M. Raczkowski, M. Capello, D. Poilblanc, R. Fresard, and A. M. Oles, Phys. Rev. B 76, 140505(R) (2007). 